\title{
28 Research Square \\ Elucidating the Key Biomarkers and Immune Infiltration in Acne by Integrated Bioinformatics Analysis
}

\section{Lu Yang}

Huashan Hospital

Yan-hong Shou

Huashan Hospital

Yong-sheng Yang

Huashan Hospital

Jin-hua Xu ( $\nabla$ jinhuaxu@fudan.edu.cn )

Huashan Hospital

\section{Research Article}

Keywords: Acne, Bioinformatics analyses, Gene Expression Omnibus, Immune cells, CIBERSORT

Posted Date: February 22nd, 2021

DOI: https://doi.org/10.21203/rs.3.rs-209484/v1

License: (c) (i) This work is licensed under a Creative Commons Attribution 4.0 International License. Read Full License 


\section{Abstract \\ Background}

Acne vulgaris is a common inflammatory condition of skin. However, the landscape of immune infiltration in acne has not been entirely described.

\section{Objectives}

This study used a bioinformatics approach to investigate the inflammatory acne-related key biomarkers and signaling pathways, and immune infiltration in the acne lesion.

\section{Methods}

Two microarray datasets (GSE108110 and GSE53795) were downloaded from Gene Expression Omnibus. We used "limma" package from R software to identify the differentially expressed genes (DEGs) and perform the functional enrichment analyses. Then we built a protein-protein interaction network (PPI), performed the hub genes' identification through STRING and Cytoscape. We applied the CIBERSORT algorithm to describe the immune infiltration in acne, and explored the correlation between biomarkers and immune infiltration. In the end, our findings in the study were verified by analyzing microarray dataset GSE6475.

\section{Results}

The differentially expressed genes (DEGs) including 292 upregulated genes and 150 downregulated genes in acne compared with non-lesional skin. The hub genes FPR1, C3AR1, CXCL1, CXCL8, FPR2, C3, CCR7, ITGB2 and pivotal pathways JAK-STAT signaling pathway, Toll-like receptor and NOD-like receptor signaling pathway were the most significantly associated with raising neutrophils, monocytes, activated mast cells, as well as reducing resting mast cells and Tregs.

\section{Conclusions}

Our study provides new insights into the pathogenesis and the targets which might be immunomodulatory potential for acne.

\section{Introduction}

Acne vulgaris (often called acne) is one of the most common inflammatory cutaneous disorders among adolescents and young adults which affects the pilosebaceous unit(1), and could lead to depression, low 
self-esteem and have adverse influences on quality of life. In adolescents, it is estimated that the incidence of acne ranges from about $40-90 \%(2,3)$. The incidence of acne has been estimated to be $9.4 \%$ by the Global Burden of Disease project, ranking it as the eighth most frequent disease worldwide(4). The skin lesions predominantly present on the face manifestating as the formation of papules, comedones, nodules, pustules and cysts but can also demonstrate on the neck, back and chest. Approximately $20 \%$ of the patients will develop severe acne, accompanied by scar formation(5).

Acne is often triggered by overgrowth of Corynebacterium acnes (C. acnes), Staphylococcus epidermidis and Malassezia furfur in the pilosebaceous units resulting in inflammation, associated with the increased sebum secretion caused by the circulating dehydroepiandrosterone (DHEA) and an abnormal hyperproliferation of follicular epidermis. In addition, plenty of risk factors for acne have been identified, including genetic factors, immune response, lifestyle factors such as eating habits (overeating dairy products, junk food), smoking, and the usage of oil-based cosmetics $(2,5)$. The processes of acne inflammation evoked intense investigation recently and both innate immunity and adaptive immunity are involved in. In terms of innate immunity, C. acnes-induced Toll-like receptor (TLR) 2 mediate the inflammation of acne(6). It has been suggested that interleukin (IL)-1a could initiate microcomedos(7) and in monocytic cells the NOD-like receptor 3 (NLRP3) inflammasome could be activated by C. acnes resulting in the IL-1 $\beta$ production (8). Besides innate immunity, adaptive immunity also participates in the inflammatory response in acne. The activation of IL-17/Th17 pathway has been found in skin tissue of acne (9). Recruitment of Th1/Th17 axis was found in clinically early acne lesions(10).Other reports showed the increased expression of cytokines, chemokines and other inflammatory markers such as TNF-a, IFN-y, IL-12, CXCL8, CXCL5, matrix metalloproteinases (MMPs), macrophage inhibitory factor (MIF) and myeloperoxidase (MPO) were found in acne lesions in vivo or non-invasive tape stripping(11, 12).

However, the etiology and pathogenesis of acne have not been completely determined. In recent years, much has been learned about immune cell infiltration in the acne's occurrence and development. The infiltration of IL-17 producing cells and elevated forkhead box P3 (FOXP3) expression have been found in acne lesions indicating a loss of $\mathrm{FOXP}^{+}$cells function deficiency $(13,14)$. The impact of psychological stress on acne has been mentioned and the release of substance $P$ from skin-expressed neurons under psychological stress could influence mast cell activities(15). The inflammatory response to C. acnes promotes the development of large numbers of neutrophils and $\mathrm{T}$ lymphocytes(16) around acne lesions. Conventional dendritic cells (cDC1)-derived vascular endothelial growth factor a (VEGF-a) is important for neutrophil recruitment and functions in the $\mathrm{C}$. acnes infected skin(17). However, the characteristic of immune infiltration in acne has not been fully elucidated, particularly in cells with a low abundance. Describing the components of immune cells infiltration are extremely meaningful for investigating the immunopathogenesis of acne and exploring novel targets for acne immunotherapeutic.

CIBERSORT is a computational approach that assesses the immune cell population profiles from fresh, frozen and fixed specimens(18) originating from RNA-seq or microarray data, which is widely used in the analysis of the infiltration of 22 kinds of immune cells in different diseases such as osteoarthritis $(19,20)$, 
ischemia-reperfusion injury in renal transplantation(21), lupus nephritis(22) and atopic dermatitis(23). However, there were few researches investigating immune cell infiltration through CIBERSORT in acne so far.

In the present study, the microarray datasets of acne was acquired from the Gene Expression Omnibus (GEO) database and then we analyzed the DEGs, identified the hub genes and GSEA, GSVA screened pivotal KEGG pathways in acne. Next, we obtained the characteristic of acne skin tissue's immune infiltration through CIBERSORT algorithm for the first time. We hope that through our research, the crucial biomarkers, pathways and immune cells infiltration in the acne could be clarified, adding extra information on the immunopathogenesis of acne.

\section{Materials And Methods}

\subsection{Microarray Data}

Gene expression profiles of GSE108110 and GSE53795 were downloaded from GEO database (https://www.ncbi.nlm.nih.gov/geo/) (GPL570 platform, Affymetrix Human Genome U133 Plus 2.0 Array), we chose 36 samples in GSE108110 including 18 acne patients' biopsies in papule less than 48 hour (h) old and 18 biopsies in their non-lesional skin. GSE53795 contains 24 samples, including 12 biopsies in papule less than $48 \mathrm{~h}$ old and biopsies in their non-lesional skin. All specimens were taken from the back. We used Bioconductor packages (http://www.bioconductor.org/) and R software (version 3.6.3; https://www.r-project.org/) in the data analyses. Raw data of GSE108110 and GSE53795 were read through the "affy" package(24), the inter-batch difference was removed using the "sva" package(25). We visualized the effect of removing inter-batch difference in the quantile-quantile plot ( $Q-Q$ plot), then we preprocessed and normalized the merged data and a three-dimensional PCA cluster plot was drawn by the "scatterplot3d" package to show the effect of inter-sample correction.

\subsection{Identification of DEGs}

We used the "limma" package to screen the DEGs(26), and then drew a volcano map of DEGs using the "ggplot2" package(27), and the "gplots" package(28) was used to draw a heatmap to show the differential expression of DEGs. The statistically significant DEGs were considered according to adjusted $P$-value $<0.05$ and $|\log 2 \mathrm{FC}|>1$. The adjusted $P$-value was corrected by the Benjamini \& Hochberg false discovery rate (FDR) method.

\subsection{PPI Network Establishment and Hub Genes Identification}

We analyzed the DEGs with the online website STRING (https://string-db.org/, version 11), 0.900 (highest confidence) was regarded as the minimum required interaction score. Subsequently, we used software Cytoscape (Version 3.7.2) to build a PPI network. CytoHubba plugin was used to determine the hub genes by integrating 12 algorithms' results. 


\subsection{Gene Set Enrichment Analyses}

GSVA was applied through the "GSVA"(29) and the "limma" packages to analyze dataset for KEGG enriched pathways with enrichment score change $>1.15$ and adjusted $P$-value $<0.05$. GSEA was performed by the iBio Tools v 5.0 by Chris Lou (www.chrislifescience.club:3838/R/AnnoE2) to identify KEGG enriched pathways in acne. $P$-value $<0.05$ and FDR $<0.25$ were considered significant enrichment.

\subsection{Evaluation of Immune Cell Infiltration by CIBERSORT Analysis}

The normalized gene expression data was uploaded to CIBERSORT, chose the samples with $P$-value $<$ 0.05 , and obtained the 22 kinds of immune cells' composition. These immune cells included naive CD $4+$ $T$ cells, activated memory CD $4+T$ cells, resting memory CD $4+T$ cells, CD8 + T cells, regulatory $T$ cells (Tregs), follicular helper T cells, gamma delta T cells, naive B cells, plasma cells, memory B cells, M0 macrophages, M1 macrophages, M2 macrophages, activated dendritic cells, resting dendritic cells, activated mast cells, resting mast cells, activated NK cells, resting NK cells, monocytes, neutrophils and eosinophils. The proportion of 22 kinds of immune cells in the acne lesion and non-lesional skin tissue was reflected by the barplot. Then we used "ggplot2" package to draw violin diagram ( $P$-value $<0.05$ was considered statistically significant) and a two-dimensional PCA map to identify if the immune cell infiltration is different between the two groups. We used the "corrplot" package(30) to visualize the correlation between immune cells; "pheatmap" package $(31,32)$ was utilized to draw a heatmap to show the different immune cell infiltration.

\subsection{Correlation Analysis between Hub Genes and Immune Cells Infiltration}

We performed Spearman correlation analysis using STATA 13.0 (College Station, Texas 77845 USA) between the abundances of 21 subpopulations of infiltrating immune cells (except eosinophils which were not infiltrated in all the skin tissues) and the expression values of hub genes, as shown in the bubble plots drawn by "ggplot2" package. $P$-value $<0.05$ was considered statistically significant.

\subsection{Correlation Analysis between Pathways and Immune Cells Infiltration}

To determine the correlation between GSEA, GSVA screened crucial pathways and infiltrating immune cells, we performed Spearman correlation analysis and linear regression analysis between the GSVA scores of pivotal KEGG pathways (including JAK-STAT signaling pathway, NOD-like receptor signaling pathway and Toll-like receptor signaling pathway) and the abundances of the infiltrated cell types, and visualized them by GraphPad Prism 8.4.2. P-value $<0.05$ was considered statistically significant.

\subsection{Validation of the Findings}


To verify our findings in this study, gene expression profiles of GSE6475 were downloaded from GEO database (https://www.ncbi.nlm.nih.gov/geo/) (GPL571 platform, Affymetrix Human Genome U133A 2.0 Array), which contained 6 acne skin samples and 6 non-lesional skin samples from acne patients, were used as a validation dataset. The same methods were utilized to analyze the data of this validation dataset. After the data preprocessed and normalized, we used the "limma" package to screen the DEGs, drew a volcano map of DEGs, then we analyzed the DEGs with the online website STRING and used software Cytoscape to build a PPI network. CytoHubba plugin was used to determine the hub genes by integrating 12 algorithms' results. Subsequently, "GSVA" and the "limma" packages were applied to analyze dataset GSE6475 for KEGG enriched pathways. In the next, we obtained 22 kinds of immune cells' composition through CIBERSORT algorithm, finally the correlation between the hub genes, crucial pathways and immune cells infiltration was analyzed (Fig. 1).

Figure 1 Graphical abstract

\section{Results}

\subsection{Preprocessing of the data and Identification of DEGs in Acne}

First, we merged the GSE108110 and GSE53795 datasets by removing the inter-batch difference between two datasets, as is shown in the Q-Q plot (Fig. 2). The results showed that the inter-batch difference had been removed. The merged data was then processed and normalized, as is presented in the threedimensional PCA cluster plots (Fig. 3A, B) before and after normalization, which explained that our sample correction obtained satisfactory result, after the sample correction, the lesional group could be distinguished from non-lesional group. Subsequently, 442 DEGs were identified in total containing 292 upregulated genes and 150 downregulated genes in lesional skin in comparison with non-lesional skin, as is shown in Fig. 3C. Figure 3D shows the DEGs expression heatmap, samples were clustered according to gene expression similarities, we can find that the acne group could be roughly distinguished from nonlesional group.

Figure 2 The Q-Q plot showed that the inter-batch difference between GSE108110 and GSE53795 datasets had been removed. The density map of the GSE53795 data distribution was expressed by the black line, the density map of the GSE108110 data distribution was expressed by the red line, the quantiles corresponding to the same cumulative probability was represented by the black circles.

Figure 3 Normalization between GSE108110 and GSE53795 datasets and the identification of DEGs. (A) The distribution of 60 samples before the sample correction, the samples of two datasets were distributed on the left and right sides respectively, but the acne lesion and non-lesional skin were not separated apparently. (B) After the sample correction, the samples were no longer clustered according to different datasets but were classified as acne group and normal group. Blue expresses the non-lesional control group and orange expresses the acne group. (C) Volcano map of DEGs; hot pink expresses up- 
regulated DEGs, grey expresses no significant DEGs, and green expresses down-regulated DEGs. (D) Heatmap of DEGs (all upregulated and downregulated genes); mediumvioletred represents acne group, violet represents the non-lesional control group. After genes clustered according to expression similarities, we can find that the acne group could be roughly distinguished from non-lesional group.

\subsection{PPI Network Construction and Hub Genes Identification}

The PPI network of the DEGs contained 905 edges and 156 nodes, including 140 upregulated genes, 16 downregulated genes (Fig. 4A). Through 12 algorithms' comprehensive analysis in cytoHubba, when the gene rank in the top 20 of nine or more algorithms, it will be selected. Because the results of each topological algorithm are different, only using one algorithm may bring inaccurate results. But if genes rank top among all or most of the algorithms, in other words, according to various topological analysis methods, these genes are most likely to be key genes, we believe that these genes can better represent the characteristics of acne, here we chose the genes ranked top in nine or more algorithms. The reason why we chose the top 20 was because through our observation, in many algorithms, the score of the $21 \mathrm{st}$ gene was significantly lower than that of the top 20, which scoring results were very close to each other, therefore, no fewer than 20 genes were selected in each algorithm. Finally, FPR1, C3AR1, CXCL1, CXCL8, FPR2, C3, CCR7 and ITGB2 were regarded as hub genes (Fig. 4B).

Figure 4 The PPI network of the DEGs. (A) PPI network of 156 DEGs was built by STRING and Cytoscape. (B) The top 8 hub genes assessed by a comprehensive analysis of 12 algorithms in cytoHubba.

\subsection{Gene Set Enrichment Analyses}

GSEA and GSVA analyses were performed by us to explore biological differences between lesional and non-lesional skin. The results proved that the JAK-STAT signaling pathway, Toll-like receptor signaling pathway and NOD-like receptor signaling pathway had more GSVA scores in acne lesion group, and less scores in non-lesional group (Fig. 5A), and genes associated with these pathways were enriched in acne lesion, but not in non-lesional skin (Fig. 5B).

Figure 5 GSEA and GSVA gene set enrichment analyses results. (A) The heatmap showed the enrichment analysis of KEGG gene set performed by GSVA scores. These KEGG gene sets were enriched in acne lesion group, including JAK-STAT signaling pathway, Toll-like receptor signaling pathway and NOD-like receptor signaling pathway. (B) GSEA gene set analysis demonstrated that genes associated with JAKSTAT signaling pathway, Toll-like receptor signaling pathway and NOD-like receptor signaling pathway were enriched in acne lesion, but not in non-lesional skin.

\subsection{Immune Infiltration Analyses}

CIBERSORT algorithm helped us obtain the abundances of 22 subpopulations of immune cells in 60 skin samples through analysis of the gene expression profile microarray data, and then we investigated the differences in immune cell infiltration between lesional and non-lesional skin. Figure $6 \mathrm{~A}$ showed the proportions of 22 subpopulations of immune cells in 60 skin tissues. Apparently, dendritic cells and $T$ cells represent the majority of all infiltrating cells. Figure 6B showed the immune cells infiltration 
heatmap. There was no eosinophil infiltrated in both groups. Compared with the non-lesional skin, neutrophils, activated mast cells, and M0 macrophages infiltrated more, whereas resting mast cells infiltrated less. PCA cluster analysis result demonstrated that the immune cell infiltration was significant different between acne lesions and normal controls (Fig. 6C). There were significant positive correlations among naïve B cells, naïve T cells, and M1 macrophages, neutrophils were positively correlated with activated mast cells, plasma cells and memory B cells also had a positive correlation. Resting mast cells were negatively correlated with activated mast cells and neutrophils (Fig. 6D). After visualizing the data using violin plot, we found that lesional skin contained a significant higher percentage of neutrophils, activated mast cells, monocytes, activated dendritic cells, M0 macrophages, resting memory CD4 + T cells, activated memory CD $4+T$ cells, while the proportions of plasma cells, memory B cells, Tregs, follicular helper T cells, resting mast cells, resting dendritic cells were relatively lower (Fig. 6E).

Figure 6 Evaluation and visualization of immune cell infiltration. (A) The relative proportion of 22 kinds of immune cells in acne (right 30 columns) and normal controls (left 30 columns). (B) Heatmap of immune cells between acne and normal controls. Blue represents acne group, red represents the normal control group. (C) PCA cluster plot showed the immune cell infiltration was significant different between acne lesions and normal controls. Red represents acne group, blue represents the normal control group. (D) Correlation heatmap explained the relevance of 21 subpopulations of immune cells (except eosinophils). The darker the color, the stronger the correlation. Blue color represents negative correlation between two cell types, red color represents positive correlation between two cell types. (E) The difference of the percentages of immune cells between acne and normal controls was illustrated by violin plot. The acne group was labeled by red color and normal control group was labeled by blue color, with a $P$-value $<0.05$ considered statistically significant.

\subsection{Correlation Analysis between Hub Genes and Infiltrating Immune Cells}

The correlation between the expression of hub genes (including FPR1, C3AR1, CXCL1, CXCL8, FPR2, C3, CCR7 and ITGB2) and the abundances of immune cells were analyzed by Spearman correlation analyses. The results showed that all these genes were positively correlated with the abundances of neutrophils, monocytes, activated mast cells, M0 macrophages, M1 macrophages, activated memory CD $4+\mathrm{T}$ cells, gamma delta $T$ cells and naive $B$ cells, and negatively correlated with the abundances of resting mast cells, Tregs, memory B cells, resting dendritic cells, follicular helper T cells and plasma cells (Fig. 7A-H).

Figure 7 Correlation between infiltrating immune cells and hub genes. $(A-H)$

\subsection{Correlation Analysis between Pathways and Infiltrating Immune Cells}

We used Spearman correlation analyses and linear regression analyses to evaluate the correlation between the GSVA scores of pivotal KEGG pathways (including JAK-STAT signaling pathway, NOD-like receptor signaling pathway and Toll-like receptor signaling pathway) and the abundances of the 
infiltrated cell types. We observed that 14 types of immune cells significantly corelated with JAK-STAT signaling pathway, NOD-like receptor signaling pathway and Toll-like receptor signaling pathway (Fig. 8A). Naive B cells, memory B cells, plasma cells, activated memory CD $4+T$ cells, Tregs, gamma delta T cells, follicular helper T cells, monocytes, M0 macrophages, M1 macrophages, resting dendritic cells, activated mast cells, resting mast cells, neutrophils were found correlated to all three pathways, among them, neutrophils and activated mast cells were most positively correlated to these pathways, resting mast cells and Tregs were most negatively correlated to all three pathways (Fig. 8B-M, Table S1).

Figure 8 Infiltrating immune cells and critical pathways' correlation. (A) The GSVA scores of JAK-STAT signaling pathway, NOD-like receptor signaling pathway, and Toll-like receptor signaling pathway were significantly correlated with the abundances of 14 types of immune cells. (B-M) Neutrophils, activated mast cells were most positively, resting mast cells and Tregs were most negatively correlated with the JAK-STAT signaling pathway, NOD-like receptor signaling pathway, and Toll-like receptor signaling pathway.

\subsection{Validation of the Findings in this Study}

To verify our findings in this study, we introduced a dataset GSE6475 as a validation dataset. In GSE6475, there were 154 up-regulated genes and 35 down-regulated genes (Fig. 9A). Among them, 116 up-regulated genes and 13 down-regulated genes were consistent with our merged data from GSE108110 and GSE53795. 4 of the top 5 up-regulated genes in the validation dataset were also ranked top 5 in our merged data, and 45 of the top 50 up-regulated genes in the validation dataset were up-regulated in our merged data. The overlapped DEGs between the validation dataset and our merged data could be seen in Table S2. The PPI network constructed by the DEGs of validation dataset was similar to our original PPI network (Fig. 9B), 7 of the 8 hub genes (including FPR1, C3AR1, CXCL1, CXCL8, FPR2, C3 and ITGB2) could be identified as hub genes in the validation dataset (Fig. 9C). There was no significant difference in the expression of CCR7 between the acne group and the non-lesional group. Through GSVA analysis, we found that similar KEGG gene sets were enriched in acne lesion group, including JAK-STAT signaling pathway, Toll-like receptor signaling pathway and NOD-like receptor signaling pathway in the validation dataset GSE6475 (Fig. 9D).

Figure 9 Screening of DEGs, and the PPI network construction as well as the gene set enrichment analyses of the DEGs in the validation dataset GSE6475. (A) Volcano map of DEGs. (B) The PPI network constructed by the DEGs of validation dataset was similar to our original PPI network. (C) 7 of the 8 hub genes (including FPR1, C3AR1, CXCL1, CXCL8, FPR2, C3 and ITGB2) could be identified as hub genes in the validation dataset. (D) The heatmap showed the enrichment analysis of KEGG gene set performed by GSVA scores in GSE6475. These KEGG gene sets were also enriched in acne lesion group, including JAKSTAT signaling pathway, Toll-like receptor signaling pathway and NOD-like receptor signaling pathway.

The abundances of 22 subpopulations of immune cells in GSE6475 showed similar characteristics to our results, PCA cluster analysis result demonstrated that the immune cell infiltration could distinguish acne lesions from normal controls (Fig. 10A), acne skin contained a higher percentage of neutrophils, activated 
mast cells (although it was not statistically significant), while Tregs and resting mast cells were relatively lower (Fig. 10B, C). Similar to our results, there were significant positive correlations between naïve B cells and M1 macrophages, neutrophils were positively correlated with activated mast cells, in addition, plasma cells and memory B cells also had a positive correlation. Resting mast cells were negatively correlated with activated mast cells and neutrophils (Fig. 10D). The hub genes FPR1, C3AR1, CXCL1, CXCL8, FPR2, C3 and ITGB2 were positively correlated with the abundances of neutrophils, monocytes, activated mast cells, M0 macrophages and activated memory CD4 + T cells, and negatively correlated with the abundances of resting mast cells, Tregs, resting dendritic cells and follicular helper T cells, which were also similar to our results (Fig. 11A-G). And we also found that mast cells, neutrophils were positively, and resting mast cells as well as Tregs were negatively correlated to all three pivotal pathways (consisting of JAK-STAT signaling pathway, Toll-like receptor signaling pathway and NOD-like receptor signaling pathway) (Fig. 12A-L). To sum up, our results from the merged data and the validation dataset remained consistent across the basic.

Figure 10 Evaluation and visualization of immune cell infiltration in the validation dataset. (A) PCA cluster plot showed the immune cell infiltration was significant different between acne lesions and normal controls. (B) The relative proportion of 22 kinds of immune cells in acne (right 6 columns) and normal controls (left 6 columns). (C) The difference of the percentages of immune cells between acne and normal controls was illustrated by violin plot. The acne group was labeled by red color and normal control group was labeled by blue color, with a $P$-value $<0.05$ considered statistically significant. (D) Correlation heatmap explained the relevance of 20 subpopulations of immune cells (except eosinophils and gamma delta T cells which were not infiltrated in both groups). The darker the color, the stronger the correlation. Blue color represents negative correlation between two cell types, red color represents positive correlation between two cell types.

Figure 11 Correlation between infiltrating immune cells and hub genes in the validation dataset. (A-G)

Figure 12 Infiltrating immune cells and critical pathways' correlation in the validation dataset.

Neutrophils, activated mast cells were positively, resting mast cells and Tregs were negatively correlated with the JAK-STAT signaling pathway, NOD-like receptor signaling pathway, and Toll-like receptor signaling pathway (A-L).

\section{Discussion}

Acne is recognized as a disease with multifactorial pathogenesis which involves the sebaceous gland, with highest prevalence among adolescents. A further understanding of the pathogenesis of this disease could provide new ideas for the mechanism-targeted treatments(33).

Inflammation has been proved to be strongly associated with the pathogenesis of acne, additionally, previous researches illustrated that immune cell infiltration is a key factor involved in the development of acne $(13,17)$. The activation of TLRs, the increased productions of chemokines for neutrophils, T cells, 
monocytes and dendritic cells, cytokines (especially Th1/Th17 related cytokines), and other inflammatory markers such as MMPs have been confirmed participating in the acne inflammation $(12,34,35)$.

Based on the GSEA and GSVA analyses, we identified that the JAK-STAT signaling pathway, NOD-like receptor signaling pathway, and Toll-like receptor signaling pathway were enriched in acne lesions. The JAK-STAT signaling pathway stimulated by cytokines, which is a signal transduction pathway(36). It plays roles in the processes of proliferation, differentiation, immunoregulation and apoptosis. According to the N-terminal domain, NOD-like receptors (NLRs) are classified into four subfamilies: NLRA, NLRB, NLRC, and NLRP(37). NLRs play a vital role in inflammasomes, $C$. acnes could powerfully trigger the activation of the NLRP3 inflammasome and the downstream caspase- 1 and IL-1 $\beta(38)$. IL-1 $\beta$ which produce the inflammatory papules of acne(39). It has been observed that NOD-like receptors could regulate the MAPK and NF-KB pathways leading to proinflammatory cytokines release such as TNF-a, IL6(40) when it acts in synergy with TLRs. Hanna-Leena Kelhälä et.al(9) observed that significant elevation of the TNF-a in acne skin compared to normal control. IL- 6 family is regarded as the key trigger of JAKSTAT pathway. J Ji et.al found that IL-6 was increased in acne patients' skin lesion tissues(41).

In our current study, we explored the crucial genes in the pathogenesis of acne, including FPR1, C3AR1, CXCL1, CXCL8, FPR2, C3, CCR7 and ITGB2. However, there was no significant difference in the expression of CCR7 between the acne group and the non-lesional group in our validation dataset. FPR1 and FPR2, the peptides belong to the formyl peptide receptor family, C3AR1, the receptor for $\mathrm{C} 3 \mathrm{a}$, members of the CXC chemokine family (such as CXCL1, CXCL8), C-C motif chemokine receptor (such as CCR2) have been identified the crucial genes in acne lesions $(42,43)$. FPR1, FPR2 and FPR3 play roles in inflammatory and antibacterial processes, which are primarily expressed in leukocytes, belonged to Gi-protein-coupled receptors. C3, C3AR1, and C5AR1 belong to the core members of complement family, participate in cellular response to inflammation(44). CXCL1, CXCL8, CXCL2, CXCL3, CXCL5 are involved in guiding the neutrophils to the site of infection and promote inflammation. CXCL9, CXCL11 and CXCL13 are chemoattractants for lymphocytes, CCR2 specifically mediates monocytes chemotaxis, CCR5 mediates monocytes, macrophages and T cells chemotaxis, and CCR7 has been proved to stimulate dendritic cell maturation as well as memory T cells' migration to inflamed tissues. ITGB2 which encodes integrin that is involved in leukocytes adhesion and cell-surface mediated signaling.

Our study not only identified a range of key genes and significant pathways in the pathogenesis of acne, but also analyzed the immune cell infiltration patterns of this disease. CIBERSORT was used to evaluate immune cell infiltration in acne. We discovered that an increased infiltration of neutrophils, activated mast cells, monocytes, activated dendritic cells, M0 macrophages, resting memory CD4 + T cells, activated memory CD $4+T$ cells, and a decreased infiltration of plasma cells, memory B cells, Tregs, follicular helper T cells, resting mast cells, resting dendritic cells may participate in the immune pathogenesis of acne.

It has been illustrated that the number of mast cells was observed to be increased in adjacent areas to the sebaceous glands in the acne lesion(45). Substance P (SP), a kind of neuropeptide associated with 
stress-induced acne, could stimulate mast cells degranulation and macrophage infiltration(15) as well as stimulate the release of a series of pro-inflammatory cytokines such as IL-6, IL-1, and TNF-a(46)from sebocytes. Hao Jiang et.al found that in acne lesions, a massive uptake of oxidized lipid could lead to the generation of neutrophils (foam cells) and lipid-loaded macrophages(47). C. acnes has been proved to activate dendritic cells and enhance the release of cytokines and adhesive molecules, leading to antigenpresenting cells that tend to activate Th17 cells(48). Min Qin et.al showed that $\mathrm{C}$. acnes stimulate monocytes to NLRP3-mediated pathway partially dependent IL-1 $\beta$ secretion(39). George W Agak et.al(49) suggested that $\mathrm{C}$. acnes strains may play different roles in skin disease and health, leading to different responses of $\mathrm{CD} 4+\mathrm{T}$ cells and either acne pathogenesis or homeostasis contributed by the generation of Th17 cells. It is well known that Th17 cells and FOXP3 + Treg cells modulate the immune balance in acne(14). I Carlavan et.al(50) found that 48-h-old papules in acne, neutrophils, T cells and macrophages infiltration increased. however, long-lived acne papules (3-week-old) in scar-prone patients are characterized by B-cell infiltration increased. Our results are consistent with the previous studies that activated mast cells, monocytes, activated memory CD4 + T cells, resting memory CD $4+T$ cells, Tregs, activated dendritic cells, M0 macrophages, plasma cells and memory B cells may participate in acne development and further research is need. Follicular helper T cells' function in acne has not been focused on, and further experimental data are required. The specific mechanisms of the correlation among immune cells infiltration in acne require further experimental evidence.

We speculated that neutrophils, monocytes, activated mast cells were the most positively, and resting mast cells, Tregs were the most negatively correlated with the hub genes, as well as the significant correlations between these immune cells with JAK-STAT signaling pathway, Toll-like receptor signaling pathway and NOD-like receptor signaling pathway. These results suggested that neutrophils, monocytes, resting mast cells, activated mast cells, Tregs may play critical roles in the occurrence and progress of acne. Further research is required to elucidate the association between genes, pathways and immune infiltration in the pathogenesis of acne.

We used CIBERSORT algorithm to identify acne lesions' immune infiltration for the first time. Our study has also some limitations. CIBERSORT analysis may deviate from phenotypic plasticity, heterotypic interactions of cells or disease-induced disorders with limited genetic data. Besides, although some of our analysis data is consistent with the previous view, and because of the difficulty in collecting skin samples from acne patients, we used GSE6475 as a validation dataset and got similar findings, which may make the conclusions of our study more convincing, using another dataset to verify the research conclusions is not as reliable as the validation in patient samples, candidate genes and pathways should be confirmed at the level of protein with the identification of the cell types expressing/producing those.

\section{Conclusions}

In the pathogenesis of acne, FPR1, C3AR1, CXCL1, CXCL8, FPR2, C3, CCR7, ITGB2 may serve as potential biomarkers in acne. However, there was no significant difference in the expression of CCR7 between the acne group and the non-lesional group in our validation dataset. Whether CCR7 can be regarded as a 
biomarker of acne remains to be verified. We found that neutrophils, activated mast cells, monocytes, activated dendritic cells, M0 macrophages, resting memory CD4 + T cells, activated memory CD $4+T$ cells, Tregs, plasma cells, memory B cells may participate in acne development and further research is need. The key genes FPR1, C3AR1, CXCL1, CXCL8, FPR2, C3, CCR7, ITGB2 and pathways such as JAK-STAT signaling pathway, Toll-like receptor signaling pathway, and NOD-like receptor signaling pathway were the most significantly associated with raising neutrophils, monocytes, activated mast cells, and reducing resting mast cells, Tregs. Further clinical and experimental research are needed to investigate the immunopathogenesis of acne and explore novel targets for acne immunotherapeutic.

Declarations

\section{Declarations}

\section{Ethics approval and consent to participate}

Not applicable.

Consent for publication

Not applicable.

\section{Competing interests}

The authors declare that the research was conducted in the absence of any commercial or financial relationships that could be construed as a potential conflict of interest.

\section{Funding}

This research received no funding.

\section{Authors' contributions}

Conceptualization: Lu Yang, Yan-hong shou and Yong-sheng Yang; methodology: Lu Yang, Yan-hong shou; visualization: Lu Yang; original draft preparation, Lu Yang; review and editing: Yong-sheng Yang; supervision: Jin-hua Xu and Yong-sheng Yang. All authors read and approved the present version and agreed to publish the manuscript.

\section{Acknowledgments}


Lu Yang and Yan-hong Shou have equal contributions to this paper and should be treated as co-first authors. The authors thank DR. Zhi-wen Luo and DR. Yu Han for technical consulting in data processing.

\section{References}

1. Barrimi M, Bouyahyaoui Y. [Particularities of psychiatric and dermatological comorbidity among medical students in Morocco: Multicenter study]. L'Encephale. 2020.

2. Wolkenstein P, Machovcová A, Szepietowski JC, Tennstedt D, Veraldi S, Delarue A. Acne prevalence and associations with lifestyle: a cross-sectional online survey of adolescents/young adults in 7 European countries. Journal of the European Academy of Dermatology and Venereology: JEADV. 2018;32(2):298-306.

3. Babar O, Mobeen A. Prevalence and Psychological Impact of Acne Vulgaris in Female Undergraduate Medical Students of Rawalpindi and Islamabad, Pakistan. Cureus. 2019;11(9):e5722.

4. Tan JK, Bhate K. A global perspective on the epidemiology of acne. The British journal of dermatology. 2015;172 Suppl 1:3-12.

5. Sutaria AH, Masood S, Schlessinger J. Acne Vulgaris. StatPearls. Treasure Island (FL): StatPearls Publishing

6. Copyright (c) 2020, StatPearls Publishing LLC.; 2020.

7. Zeng R, Xu H, Liu Y, Du L, Duan Z, Tong J, et al. miR-146a Inhibits Biofilm-Derived Cutibacterium acnes-Induced Inflammatory Reactions in Human Keratinocytes. The Journal of investigative dermatology. 2019;139(12):2488-96.e4.

8. Thiboutot DM. Inflammasome activation by Propionibacterium acnes: the story of IL-1 in acne continues to unfold. The Journal of investigative dermatology. 2014;134(3):595-7.

9. Kistowska M, Gehrke S, Jankovic D, Kerl K, Fettelschoss A, Feldmeyer L, et al. IL-1 $\beta$ drives inflammatory responses to propionibacterium acnes in vitro and in vivo. The Journal of investigative dermatology. 2014;134(3):677-85.

10. Kelhälä HL, Palatsi R, Fyhrquist N, Lehtimäki S, Väyrynen JP, Kallioinen M, et al. IL-17/Th17 pathway is activated in acne lesions. PloS one. 2014;9(8):e105238.

11. Sardana K, Verma G. Propionibacterium acnes and the Th1/Th17 Axis, Implications in Acne Pathogenesis and Treatment. Indian journal of dermatology. 2017;62(4):392-4.

12. Dréno $B$. What is new in the pathophysiology of acne, an overview. Journal of the European Academy of Dermatology and Venereology: JEADV. 2017;31 Suppl 5:8-12.

13. Méhul B, Séraïdaris A, Blanchet-Réthoré S, Gamboa B, Bahadoran P, Queille-Roussel C, et al. Noninvasive profiling for cytokines, chemokines and growth factors in acne vulgaris. Journal of the European Academy of Dermatology and Venereology: JEADV. 2019;33(10):e386-e8.

14. Abd-Elmaged WM, Nada EA, Hassan MH, Elsadek BEM, Abdelrahim EA, Ahmed NS, et al. Lesional and circulating levels of interleukin-17 and 25-hydroxycholecalciferol in active acne vulgaris: Correlation to disease severity. Journal of cosmetic dermatology. 2019;18(2):671-6. 
15. Farag AGA, Maraee AH, Rifaat Al-Sharaky D, Elshaib ME, Kohla MSM, Shehata WA. Tissue expression of IL-17A and FOXP3 in acne vulgaris patients. Journal of cosmetic dermatology. 2020.

16. Pondeljak N, Lugović-Mihić L. Stress-induced Interaction of Skin Immune Cells, Hormones, and Neurotransmitters. Clinical therapeutics. 2020;42(5):757-70.

17. Kistowska M, Meier B, Proust T, Feldmeyer L, Cozzio A, Kuendig T, et al. Propionibacterium acnes promotes Th17 and Th17/Th1 responses in acne patients. The Journal of investigative dermatology. 2015;135(1):110-8.

18. Janela B, Patel AA, Lau MC, Goh CC, Msallam R, Kong WT, et al. A Subset of Type I Conventional Dendritic Cells Controls Cutaneous Bacterial Infections through VEGFa-Mediated Recruitment of Neutrophils. Immunity. 2019;50(4):1069-83.e8.

19. Newman AM, Liu CL, Green MR, Gentles AJ, Feng W, Xu Y, et al. Robust enumeration of cell subsets from tissue expression profiles. Nature methods. 2015;12(5):453-7.

20. Deng YJ, Ren EH, Yuan WH, Zhang GZ, Wu ZL, Xie QQ. GRB10 and E2F3 as Diagnostic Markers of Osteoarthritis and Their Correlation with Immune Infiltration. Diagnostics (Basel, Switzerland). 2020;10(3).

21. Cai W, Li H, Zhang Y, Han G. Identification of key biomarkers and immune infiltration in the synovial tissue of osteoarthritis by bioinformatics analysis. PeerJ. 2020;8:e8390.

22. Zhang J, Wei X, Tang Z, Miao B, Luo Y, Hu X, et al. Elucidating the molecular pathways and immune system transcriptome during ischemia-reperfusion injury in renal transplantation. International immunopharmacology. 2020;81:106246.

23. Cao Y, Tang W, Tang W. Immune cell infiltration characteristics and related core genes in lupus nephritis: results from bioinformatic analysis. BMC immunology. 2019;20(1):37.

24. Félix Garza ZC, Lenz M, Liebmann J, Ertaylan G, Born M, Arts ICW, et al. Characterization of diseasespecific cellular abundance profiles of chronic inflammatory skin conditions from deconvolution of biopsy samples. BMC medical genomics. 2019;12(1):121.

25. Gautier L, Cope L, Bolstad BM, Irizarry RA. affy-analysis of Affymetrix GeneChip data at the probe level. Bioinformatics (Oxford, England). 2004;20(3):307-15.

26. Parker HS, Leek JT, Favorov AV, Considine M, Xia X, Chavan S, et al. Preserving biological heterogeneity with a permuted surrogate variable analysis for genomics batch correction. Bioinformatics (Oxford, England). 2014;30(19):2757-63.

27. Ritchie ME, Phipson B, Wu D, Hu Y, Law CW, Shi W, et al. limma powers differential expression analyses for RNA-sequencing and microarray studies. Nucleic acids research. 2015;43(7):e47.

28. Wickham H. Ggplot2: Elegant Graphics for Data Analysis: Springer Publishing Company, Incorporated; 2009.

29. Warnes G, Bolker B, Bonebakker L, Gentleman R, Huber W, Liaw A, et al. gplots: Various R programming tools for plotting data2005. 
30. Hänzelmann S, Castelo R, Guinney J. GSVA: gene set variation analysis for microarray and RNA-seq data. BMC bioinformatics. 2013;14:7.

31. Friendly M. Corrgrams: Exploratory Displays for Correlation Matrices. American Statistician. 2002;56(4):316-24.

32. Szekely G, Rizzo M. Hierarchical Clustering via Joint Between-Within Distances: Extending Ward's Minimum Variance Method. Journal of Classification. 2005;22:151-83.

33. Kraskov A, Stögbauer H, Andrzejak RG, Grassberger P. Hierarchical clustering using mutual information. Europhysics Letters (EPL). 2005;70(2):278-84.

34. Ottaviani M, Flori E, Mastrofrancesco A, Briganti S, Lora V, Capitanio B, et al. Sebocyte differentiation as a new target for acne therapy: an in vivo experience. Journal of the European Academy of Dermatology and Venereology: JEADV. 2020.

35. Suvanprakorn P, Tongyen T, Prakhongcheep O, Laoratthaphong P, Chanvorachote P. Establishment of an Anti-acne Vulgaris Evaluation Method Based on TLR2 and TLR4-mediated Interleukin-8 Production. In vivo (Athens, Greece). 2019;33(6):1929-34.

36. Ozkanli S, Karadag AS, Ozlu E, Uzuncakmak TK, Takci Z, Zemheri E, et al. A comparative study of MMP-1, MMP-2, and TNF-a expression in different acne vulgaris lesions. International journal of dermatology. 2016;55(12):1402-7.

37. Morris R, Kershaw NJ, Babon JJ. The molecular details of cytokine signaling via the JAK/STAT pathway. Protein science: a publication of the Protein Society. 2018;27(12):1984-2009.

38. Kim YK, Shin JS, Nahm MH. NOD-Like Receptors in Infection, Immunity, and Diseases. Yonsei medical journal. 2016;57(1):5-14.

39. Berthelot JM, Corvec S, Hayem G. SAPHO, autophagy, IL-1, Fox01, and Propionibacterium (Cutibacterium) acnes. Joint bone spine. 2018;85(2):171-6.

40. Qin M, Pirouz A, Kim MH, Krutzik SR, Garbán HJ, Kim J. Propionibacterium acnes Induces IL-1 $\beta$ secretion via the NLRP3 inflammasome in human monocytes. The Journal of investigative dermatology. 2014;134(2):381-8.

41. Newton K, Dixit VM. Signaling in innate immunity and inflammation. Cold Spring Harbor perspectives in biology. 2012;4(3).

42. Ji J, Zhang RH, Li HM, Guo Q, Zhang LL, Zhu J, et al. Correlations of SOX9 expression with serum IGF1 and inflammatory cytokines IL-1 $\mathrm{a}$ and IL- 6 in skin lesions of patients with acne. European review for medical and pharmacological sciences. 2018;22(9):2549-55.

43. Li X, Jia Y, Wang S, Meng T, Zhu M. Identification of Genes and Pathways Associated with Acne Using Integrated Bioinformatics Methods. Dermatology (Basel, Switzerland). 2019;235(6):445-55.

44. Chen B, Zheng Y, Liang Y. Analysis of Potential Genes and Pathways Involved in the Pathogenesis of Acne by Bioinformatics. BioMed research international. 2019;2019:3739086.

45. Hawksworth OA, Li XX, Coulthard LG, Wolvetang EJ, Woodruff TM. New concepts on the therapeutic control of complement anaphylatoxin receptors. Molecular immunology. 2017;89:36-43. 
46. Toyoda M, Morohashi M. New aspects in acne inflammation. Dermatology (Basel, Switzerland). 2003;206(1):17-23.

47. Epel ES, Blackburn EH, Lin J, Dhabhar FS, Adler NE, Morrow JD, et al. Accelerated telomere shortening in response to life stress. Proceedings of the National Academy of Sciences of the United States of America. 2004;101(49):17312-5.

48. Jiang H, Li C. Common Pathogenesis of Acne Vulgaris and Atherosclerosis. Inflammation. 2019;42(1):1-5.

49. Mattii M, Lovászi M, Garzorz N, Atenhan A, Quaranta M, Lauffer F, et al. Sebocytes contribute to skin inflammation by promoting the differentiation of $\mathrm{T}$ helper 17 cells. The British journal of dermatology. 2018;178(3):722-30.

50. Agak GW, Kao S, Ouyang K, Qin M, Moon D, Butt A, et al. Phenotype and Antimicrobial Activity of Th17 Cells Induced by Propionibacterium acnes Strains Associated with Healthy and Acne Skin. The Journal of investigative dermatology. 2018;138(2):316-24.

51. Carlavan I, Bertino B, Rivier M, Martel P, Bourdes V, Motte M, et al. Atrophic scar formation in patients with acne involves long-acting immune responses with plasma cells and alteration of sebaceous glands. The British journal of dermatology. 2018;179(4):906-17.

\section{Figures}

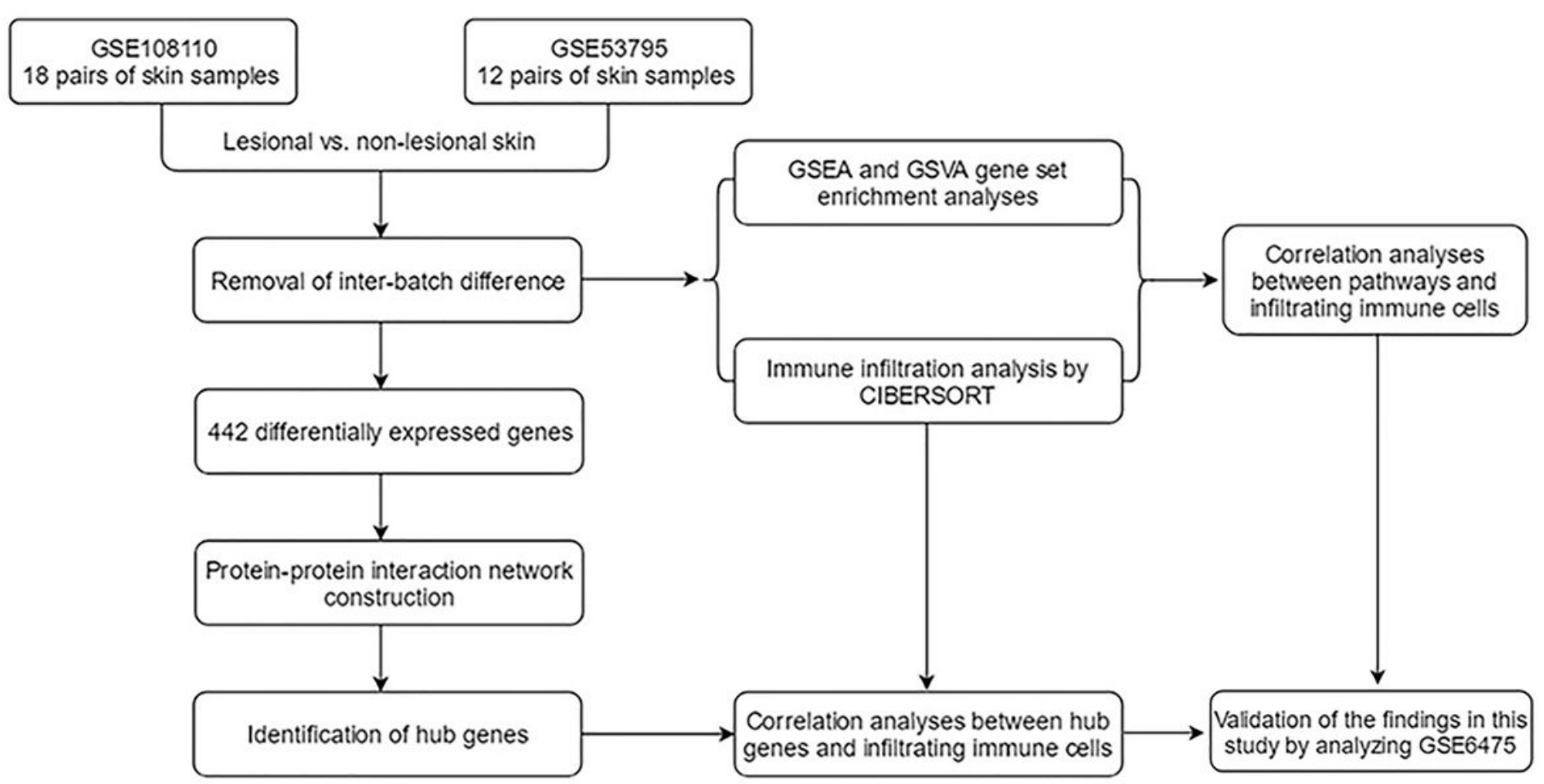

\section{Figure 1}

Graphical abstract 

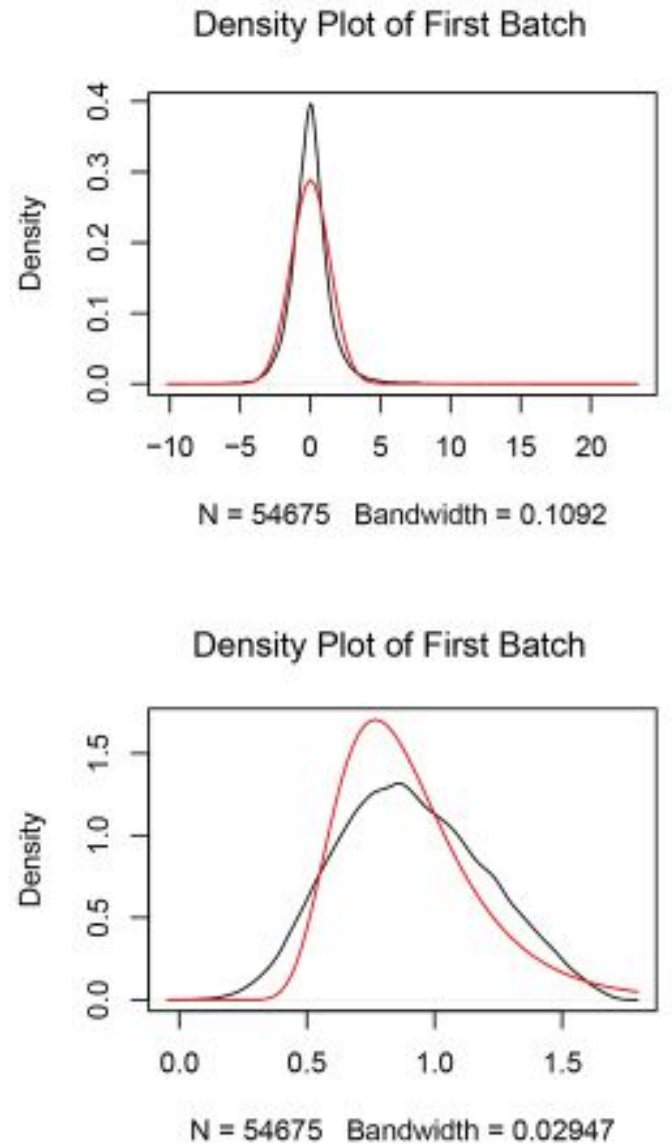

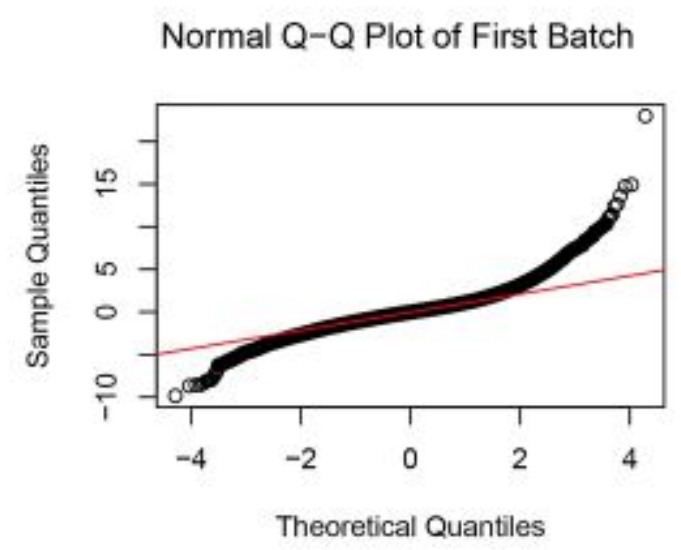

Inverse Gamma Q-Q Plot of First Batch

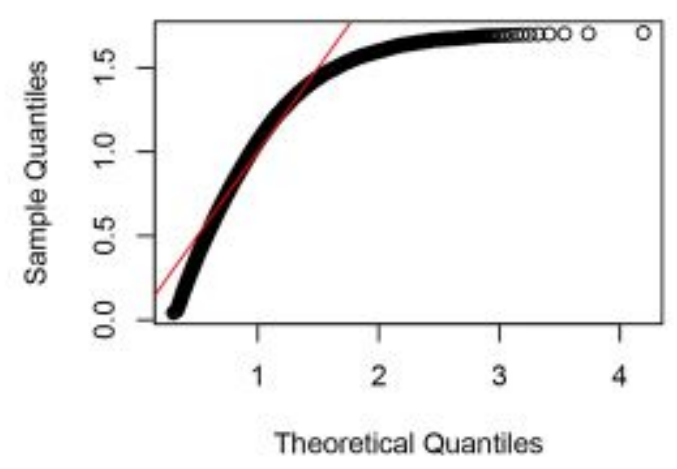

\section{Figure 2}

The Q-Q plot showed that the inter-batch difference between GSE108110 and GSE53795 datasets had been removed. The density map of the GSE53795 data distribution was expressed by the black line, the density map of the GSE108110 data distribution was expressed by the red line, the quantiles corresponding to the same cumulative probability was represented by the black circles. 
(A)

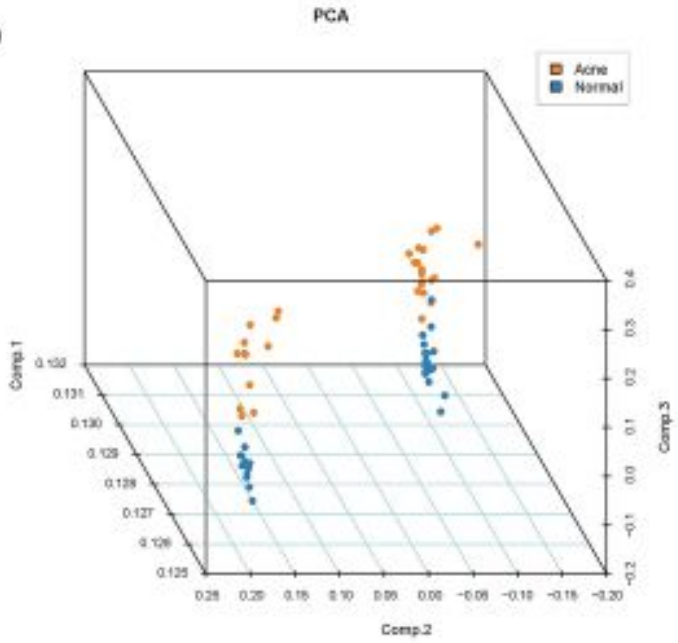

(C)

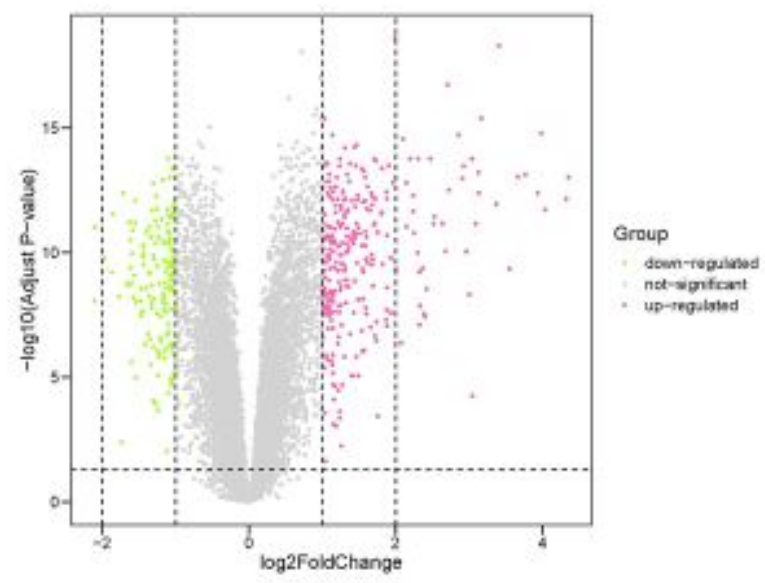

(B)

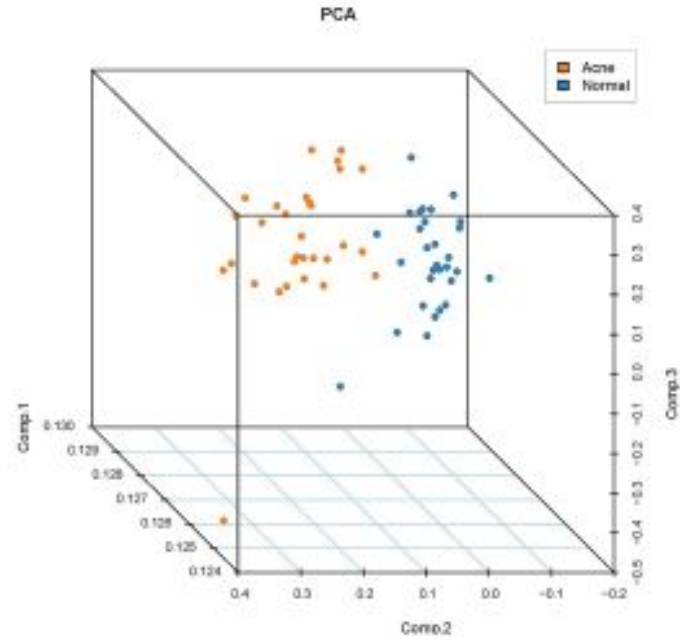

(D)
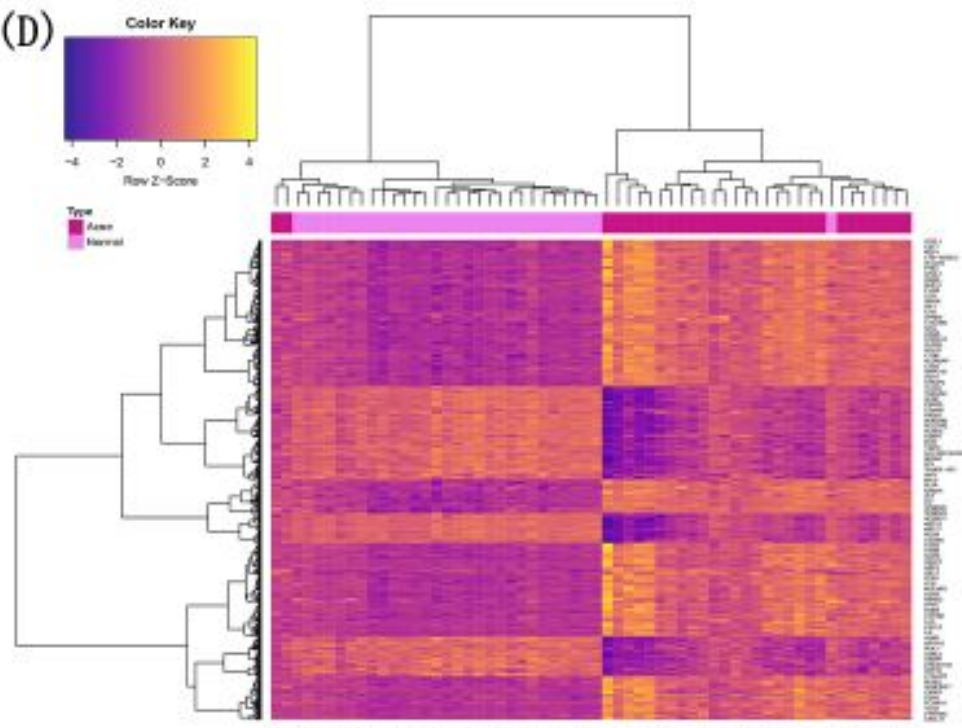

||||||||||||||||||||||||||||||||||||||||||||||||||

\section{Figure 3}

Normalization between GSE108110 and GSE53795 datasets and the identification of DEGs. (A) The distribution of 60 samples before the sample correction, the samples of two datasets were distributed on the left and right sides respectively, but the acne lesion and non-lesional skin were not separated apparently. (B) After the sample correction, the samples were no longer clustered according to different datasets but were classified as acne group and normal group. Blue expresses the non-lesional control group and orange expresses the acne group. (C) Volcano map of DEGs; hot pink expresses up-regulated DEGs, grey expresses no significant DEGs, and green expresses down-regulated DEGs. (D) Heatmap of DEGs (all upregulated and downregulated genes); mediumvioletred represents acne group, violet represents the non-lesional control group. After genes clustered according to expression similarities, we can find that the acne group could be roughly distinguished from non-lesional group. 


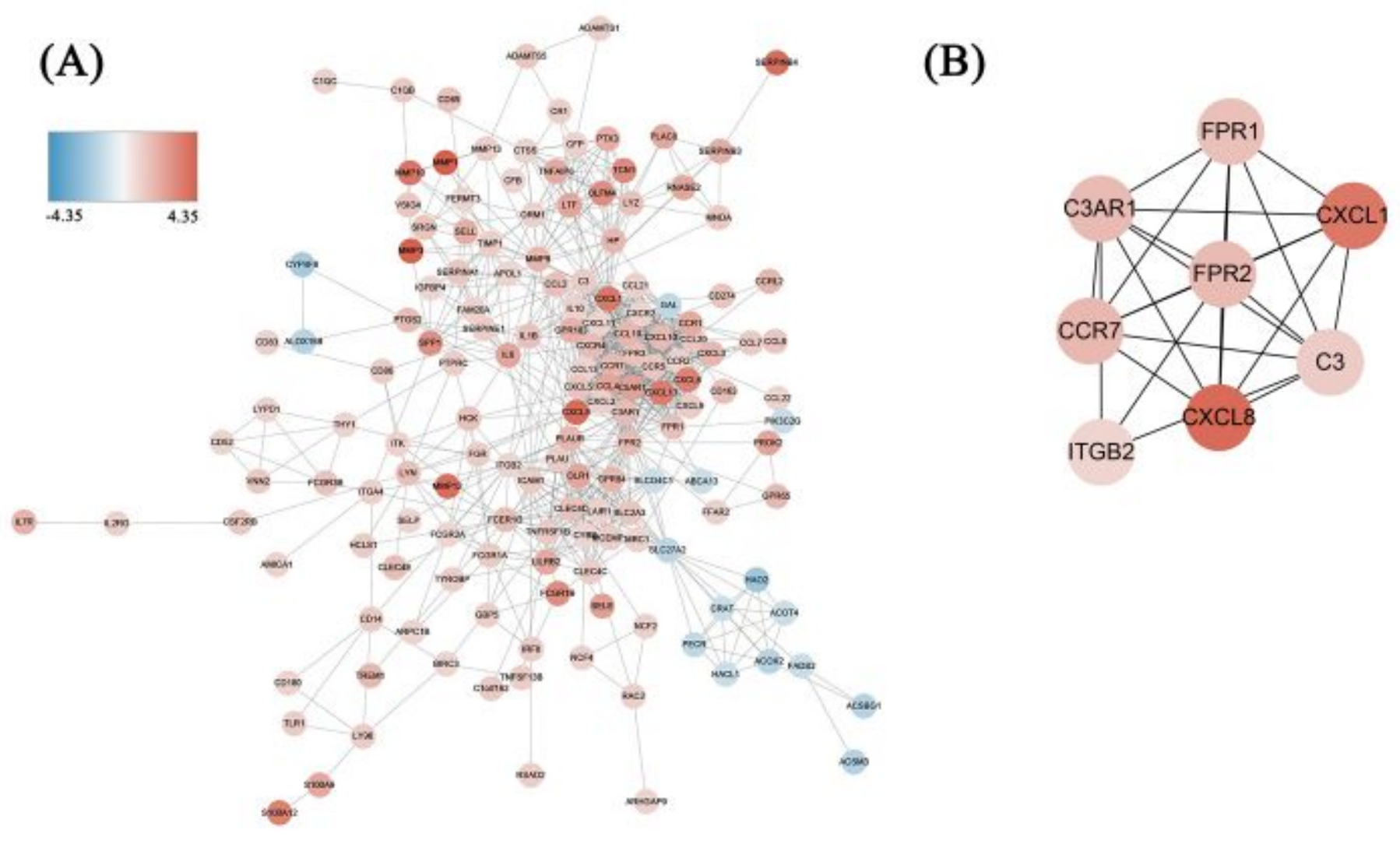

\section{Figure 4}

The PPI network of the DEGs. (A) PPI network of 156 DEGs was built by STRING and Cytoscape. (B) The top 8 hub genes assessed by a comprehensive analysis of 12 algorithms in cytoHubba. 


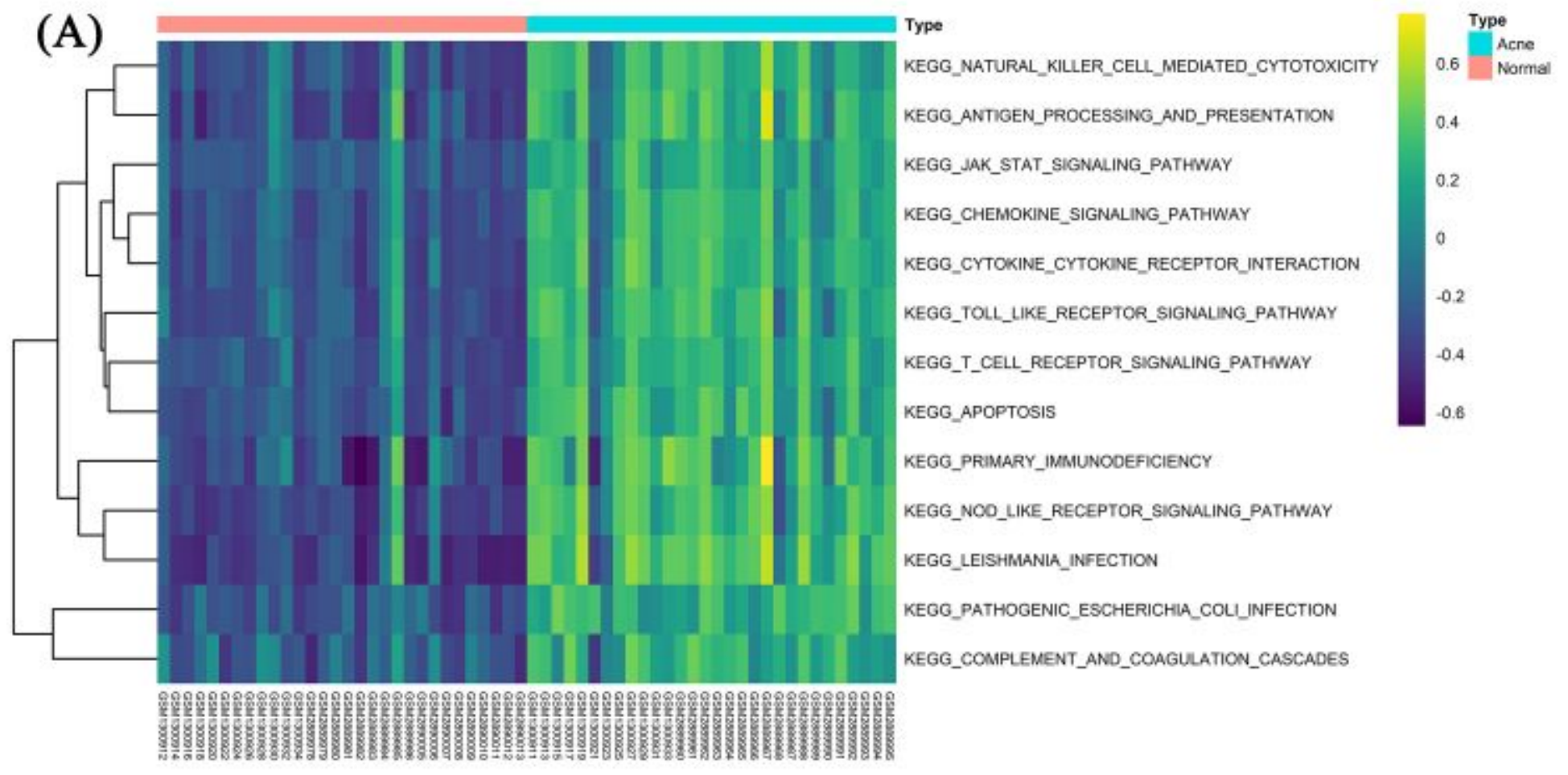

(B) - KEGG_JAK_STAT_SIGNULING_PATHWAY - KEGG_NOD_LIKE_AECEPTOR_SIONALING_PATHWAY - KEGG_TOLL_LIKE_RECEPTOR_SIONNLLING_PATHWAY

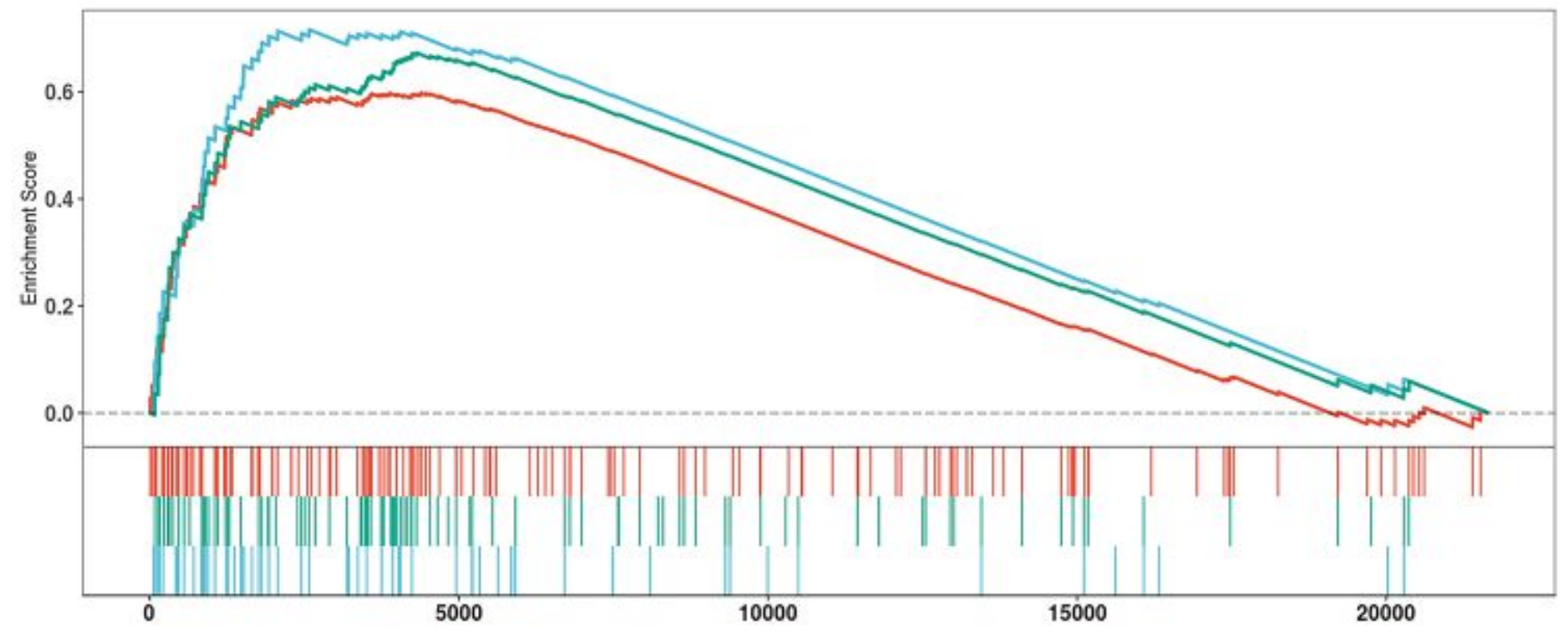

\section{Figure 5}

GSEA and GSVA gene set enrichment analyses results. (A) The heatmap showed the enrichment analysis of KEGG gene set performed by GSVA scores. These KEGG gene sets were enriched in acne lesion group, including JAK-STAT signaling pathway, Toll-like receptor signaling pathway and NOD-like receptor signaling pathway. (B) GSEA gene set analysis demonstrated that genes associated with JAK-STAT signaling pathway, Toll-like receptor signaling pathway and NOD-like receptor signaling pathway were enriched in acne lesion, but not in non-lesional skin. 
(A)

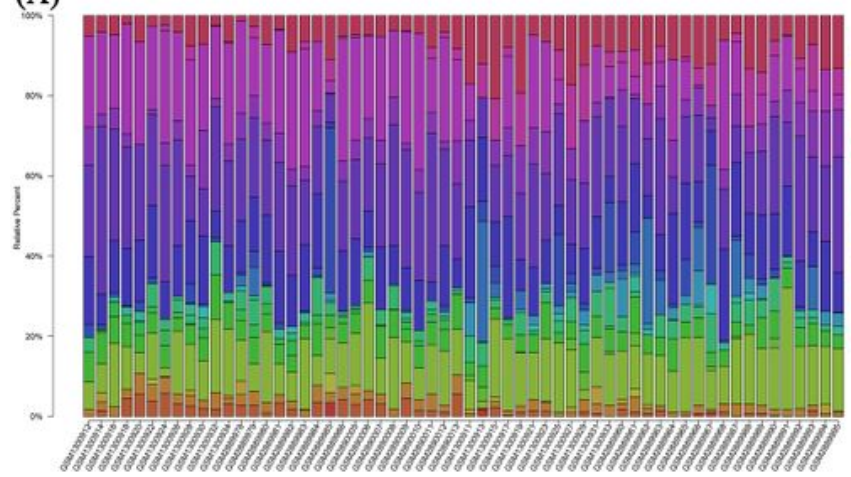

: Bcalt naivo

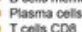

Tcosis CDA navo

cat memory actival

T colsts rogulatiory (firogs)

NK cels resting

- Macrophagos MO

Denderitc cols astivated

Nestrophis

(B)

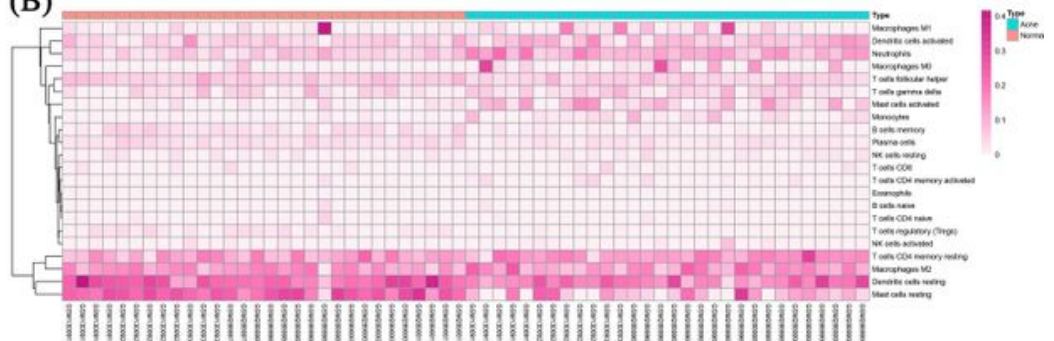

(C)

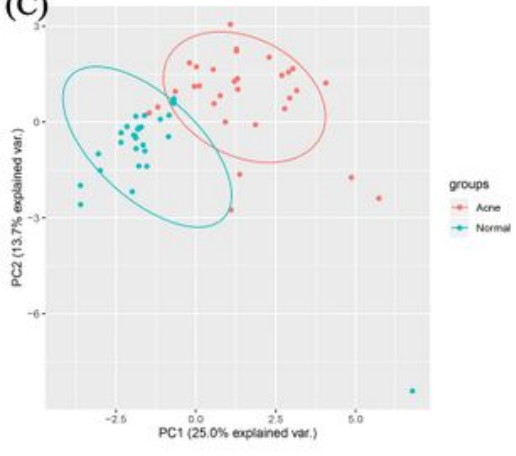

(D)

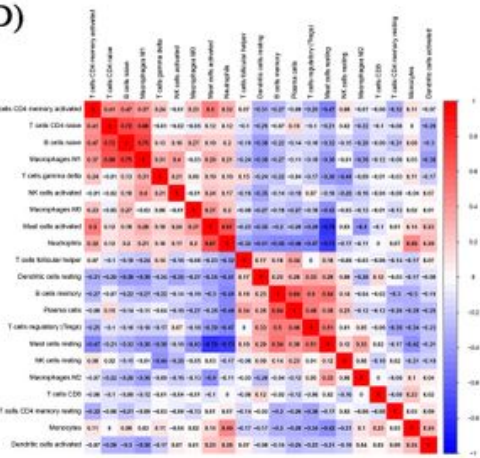

(E)

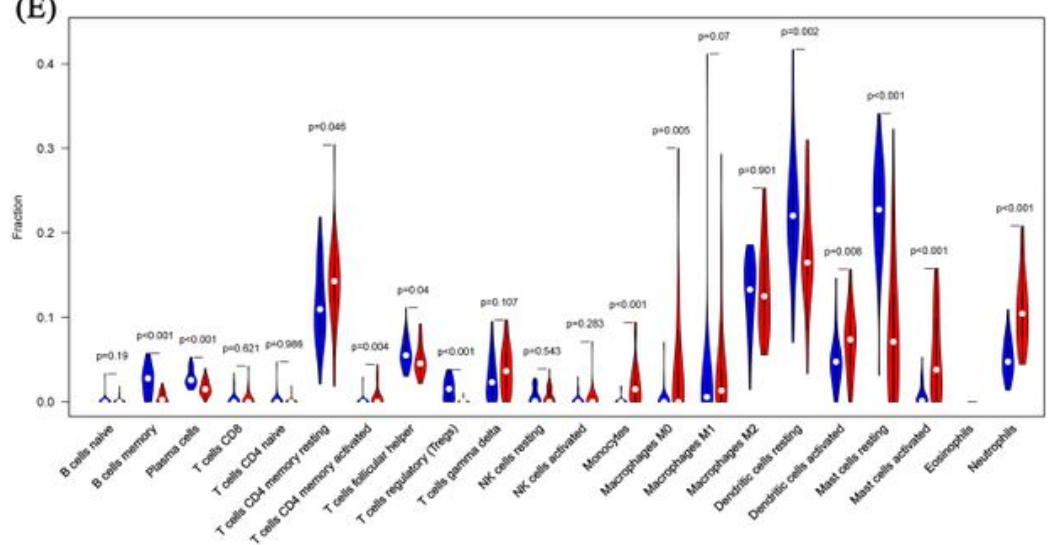

Figure 6

Evaluation and visualization of immune cell infiltration. (A) The relative proportion of 22 kinds of immune cells in acne (right 30 columns) and normal controls (left 30 columns). (B) Heatmap of immune cells between acne and normal controls. Blue represents acne group, red represents the normal control group. (C) PCA cluster plot showed the immune cell infiltration was significant different between acne lesions and normal controls. Red represents acne group, blue represents the normal control group. (D) Correlation 
heatmap explained the relevance of 21 subpopulations of immune cells (except eosinophils). The darker the color, the stronger the correlation. Blue color represents negative correlation between two cell types, red color represents positive correlation between two cell types. (E) The difference of the percentages of immune cells between acne and normal controls was illustrated by violin plot. The acne group was labeled by red color and normal control group was labeled by blue color, with a P-value $<0.05$ considered statistically significant.
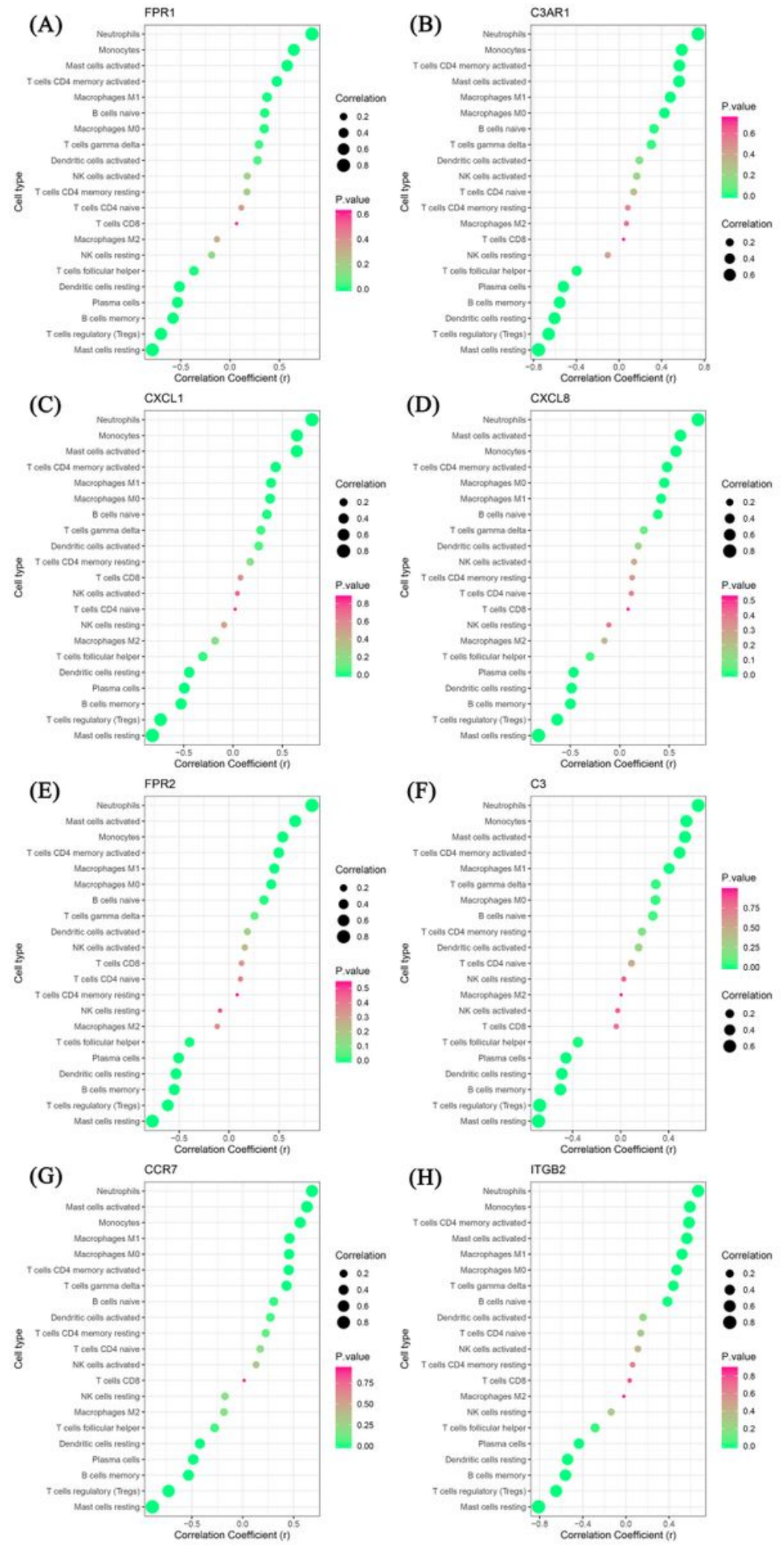

Figure 7 
Correlation between infiltrating immune cells and hub genes. $(\mathrm{A}-\mathrm{H})$

(A)

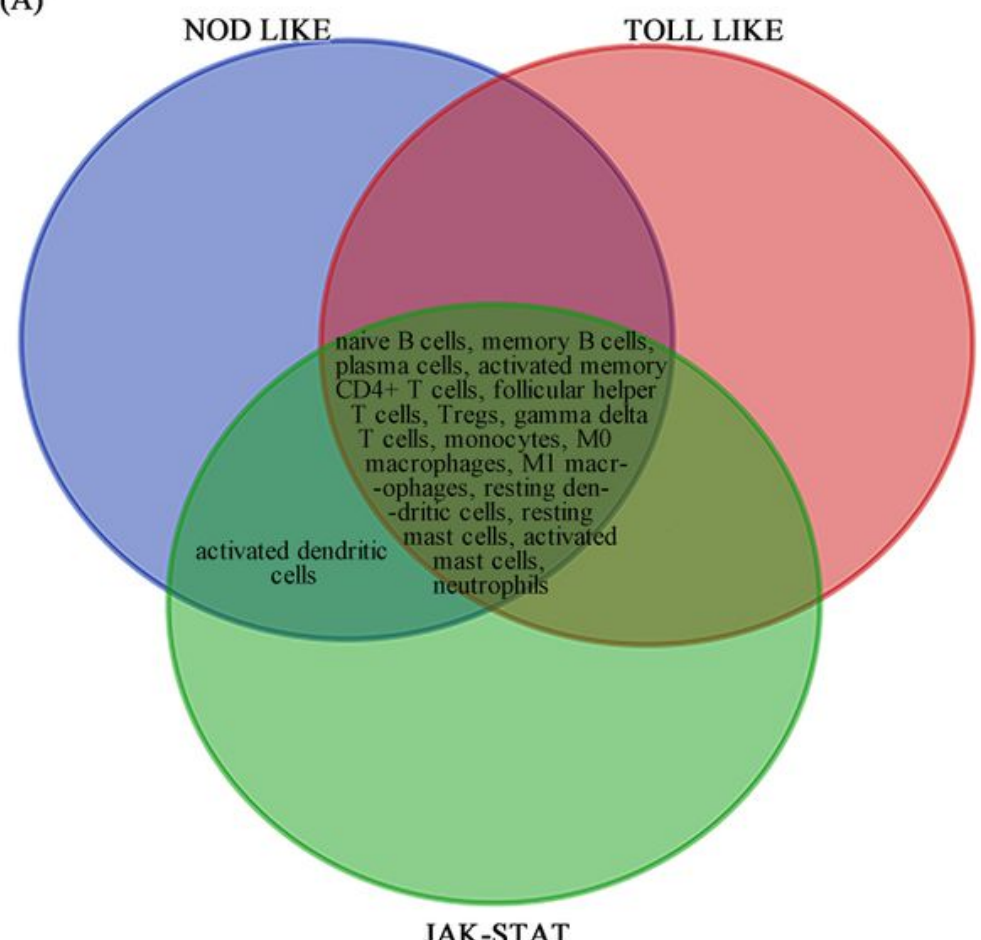

(B)

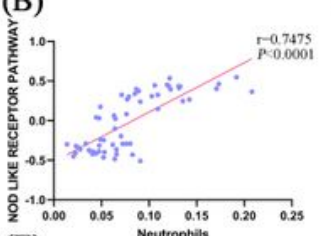

(E)

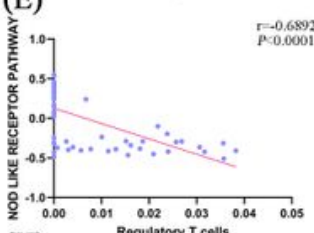

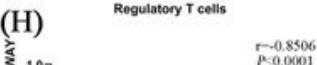
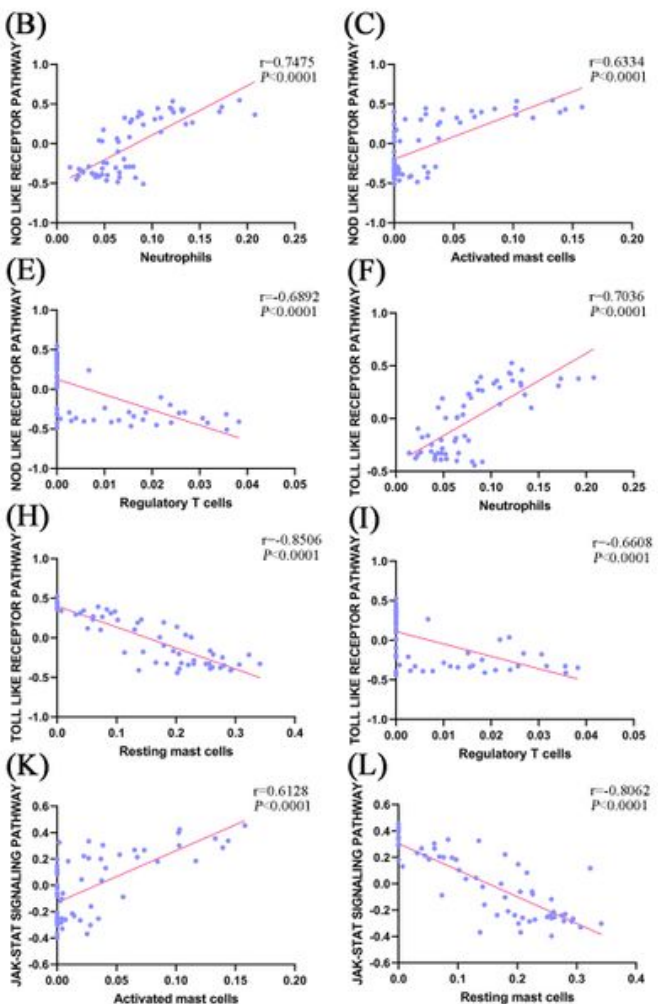

(L)

(D)

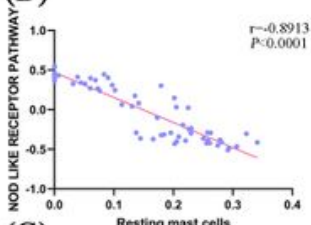

(G)
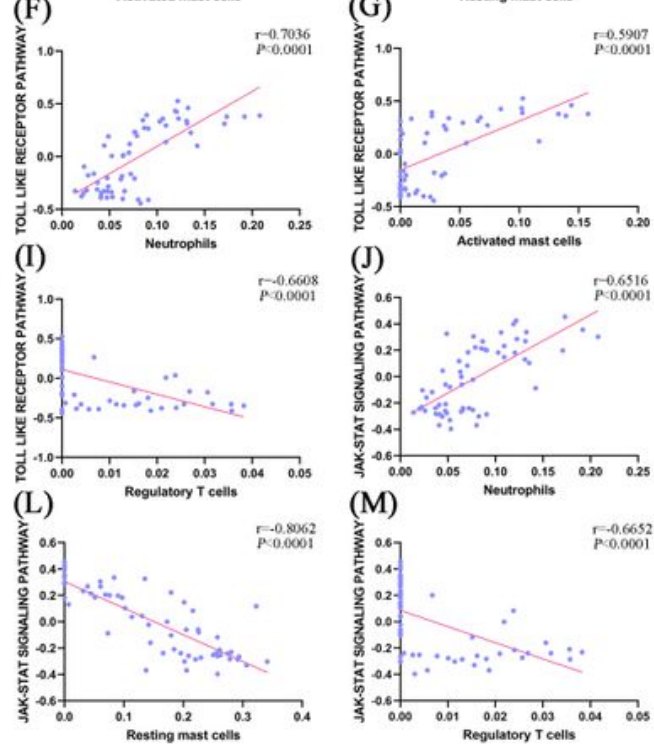

Figure 8

Infiltrating immune cells and critical pathways' correlation. (A) The GSVA scores of JAK-STAT signaling pathway, NOD-like receptor signaling pathway, and Toll-like receptor signaling pathway were significantly correlated with the abundances of 14 types of immune cells. (B-M) Neutrophils, activated mast cells 
were most positively, resting mast cells and Tregs were most negatively correlated with the JAK-STAT signaling pathway, NOD-like receptor signaling pathway, and Toll-like receptor signaling pathway.

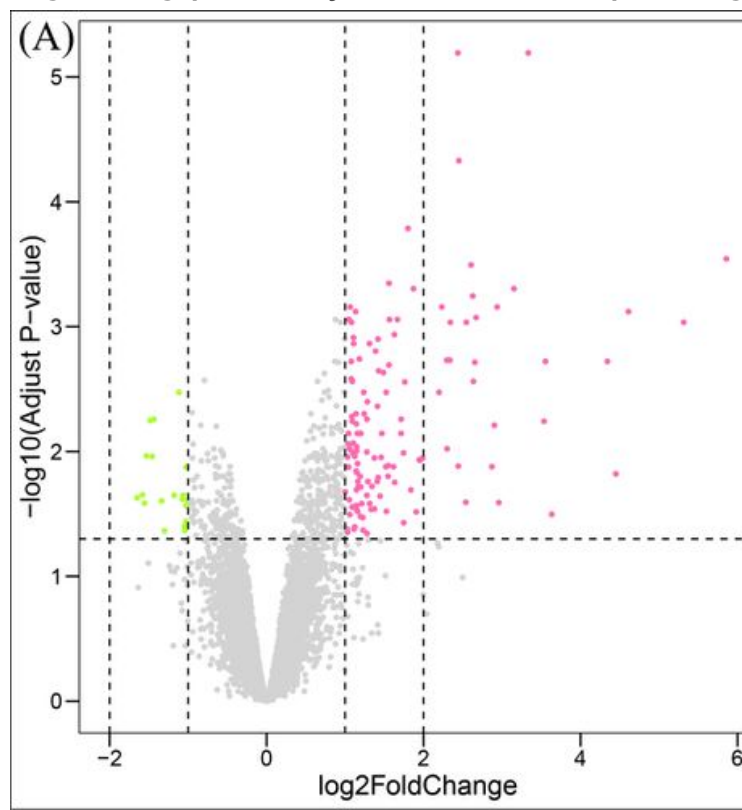

(B)
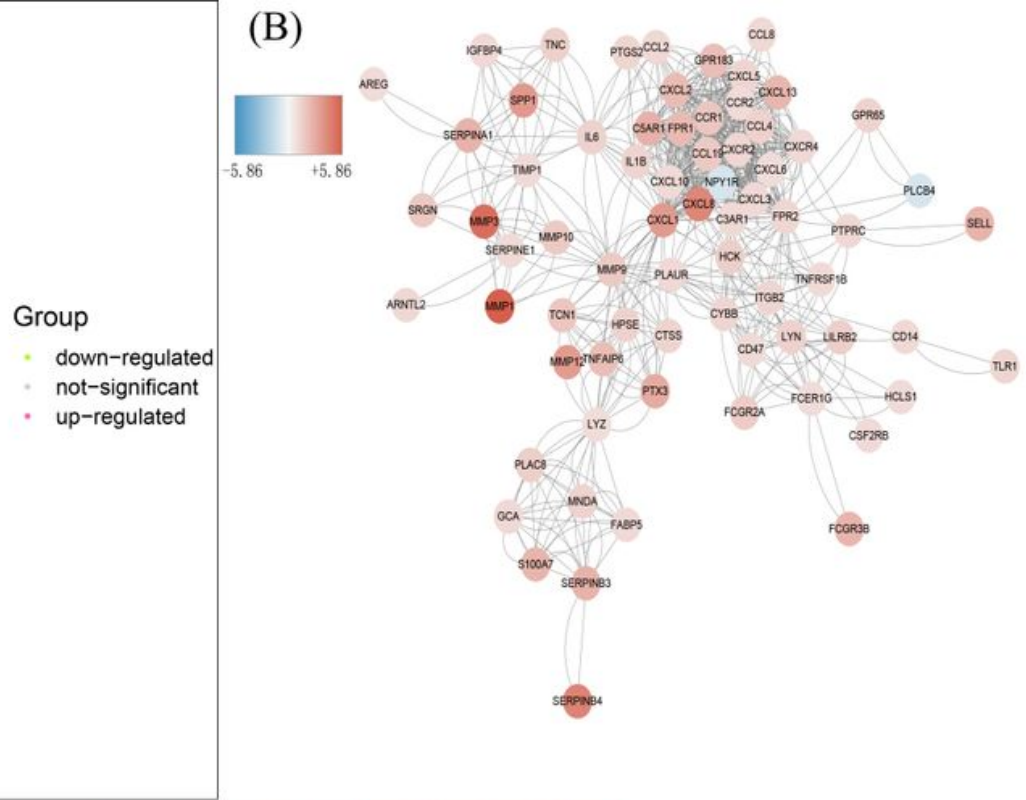

(C)
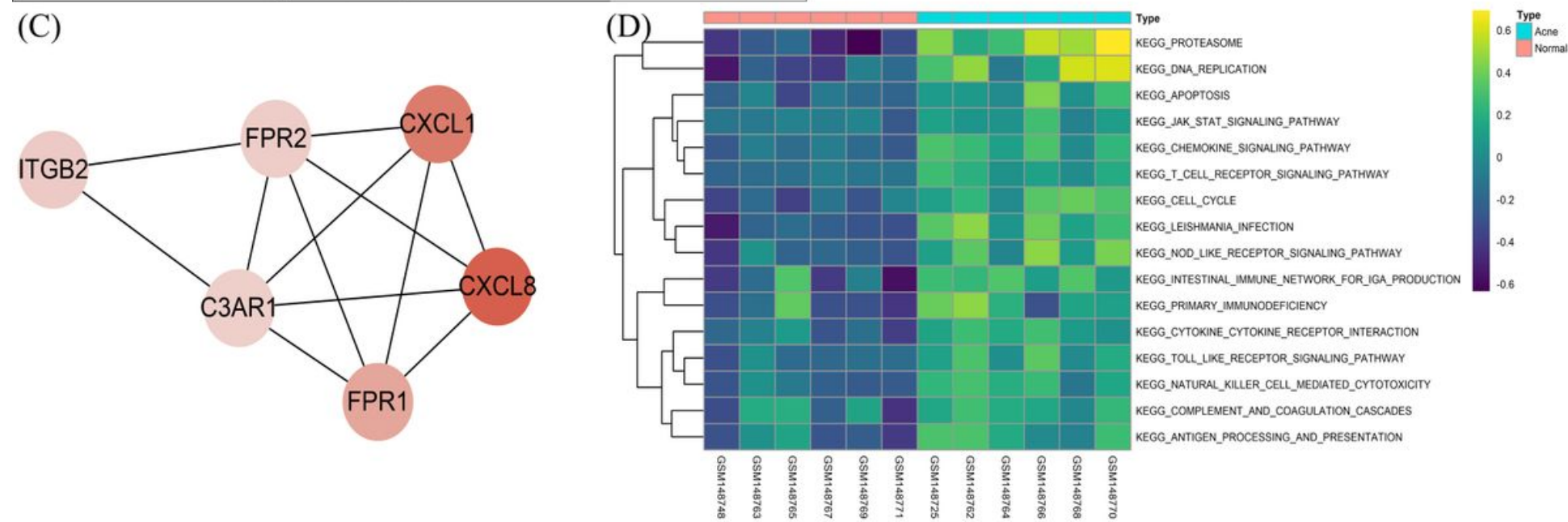

Figure 9

Screening of DEGs, and the PPI network construction as well as the gene set enrichment analyses of the DEGs in the validation dataset GSE6475. (A) Volcano map of DEGs. (B) The PPI network constructed by the DEGs of validation dataset was similar to our original PPI network. (C) 7 of the 8 hub genes (including FPR1, C3AR1, CXCL1, CXCL8, FPR2, C3 and ITGB2) could be identified as hub genes in the validation dataset. (D) The heatmap showed the enrichment analysis of KEGG gene set performed by GSVA scores in GSE6475. These KEGG gene sets were also enriched in acne lesion group, including JAK-STAT signaling pathway, Toll-like receptor signaling pathway and NOD-like receptor signaling pathway. 
(A)

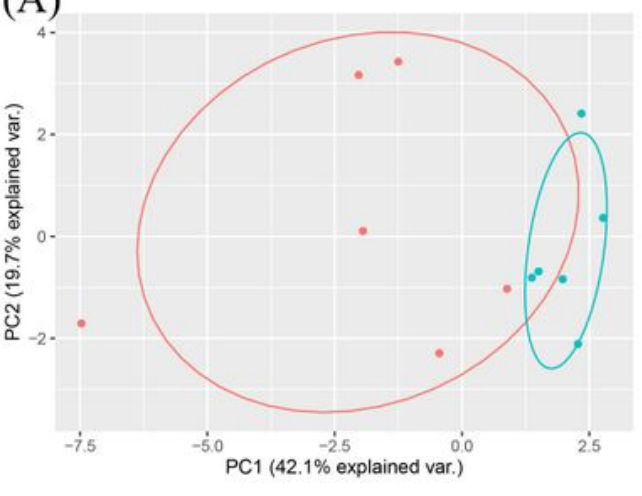

(C)
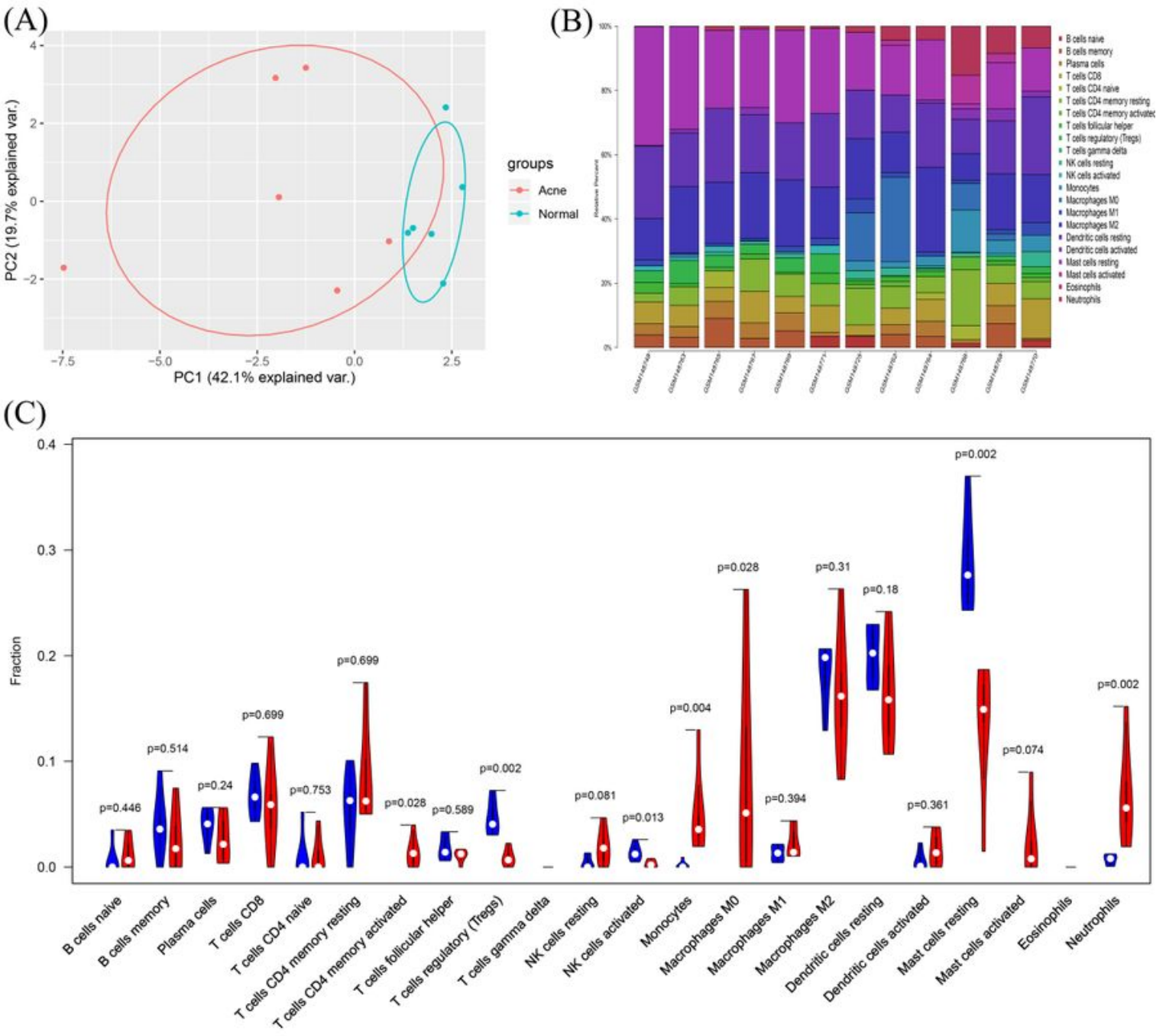

$p=0.002$

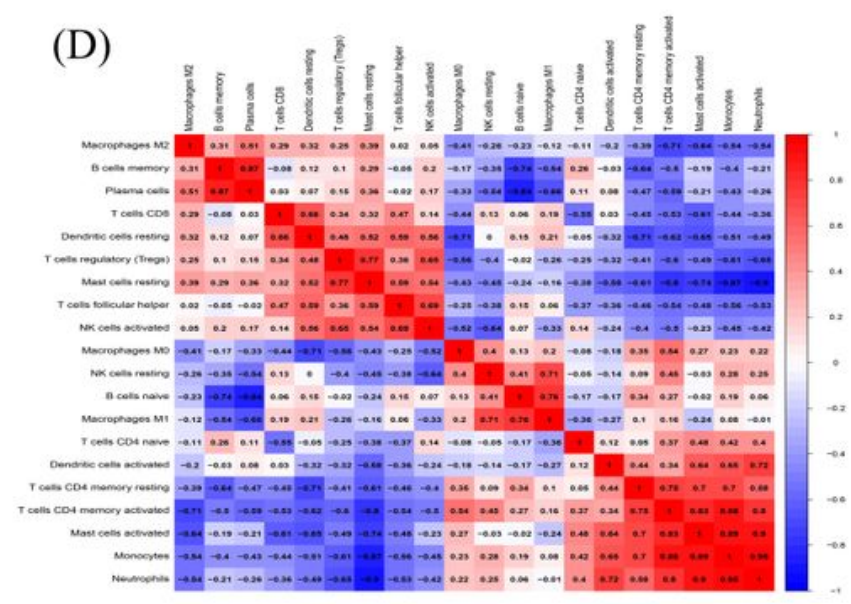

Figure 10

Evaluation and visualization of immune cell infiltration in the validation dataset. (A) PCA cluster plot showed the immune cell infiltration was significant different between acne lesions and normal controls.

(B) The relative proportion of 22 kinds of immune cells in acne (right 6 columns) and normal controls (left 6 columns). (C) The difference of the percentages of immune cells between acne and normal controls was illustrated by violin plot. The acne group was labeled by red color and normal control group was 
labeled by blue color, with a P-value $<0.05$ considered statistically significant. (D) Correlation heatmap explained the relevance of 20 subpopulations of immune cells (except eosinophils and gamma delta $T$ cells which were not infiltrated in both groups). The darker the color, the stronger the correlation. Blue color represents negative correlation between two cell types, red color represents positive correlation between two cell types.
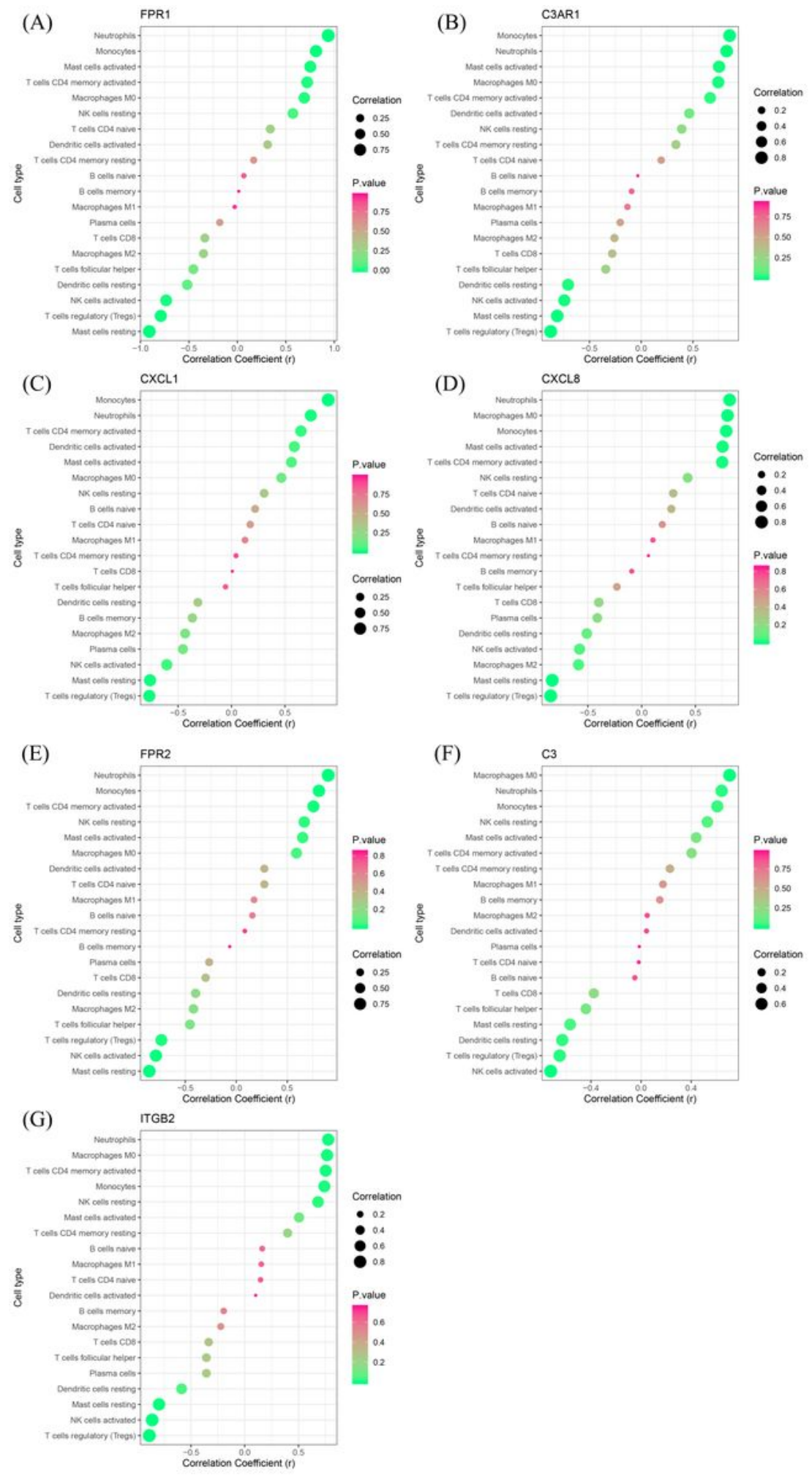

Figure 11 
Correlation between infiltrating immune cells and hub genes in the validation dataset. (A-G)
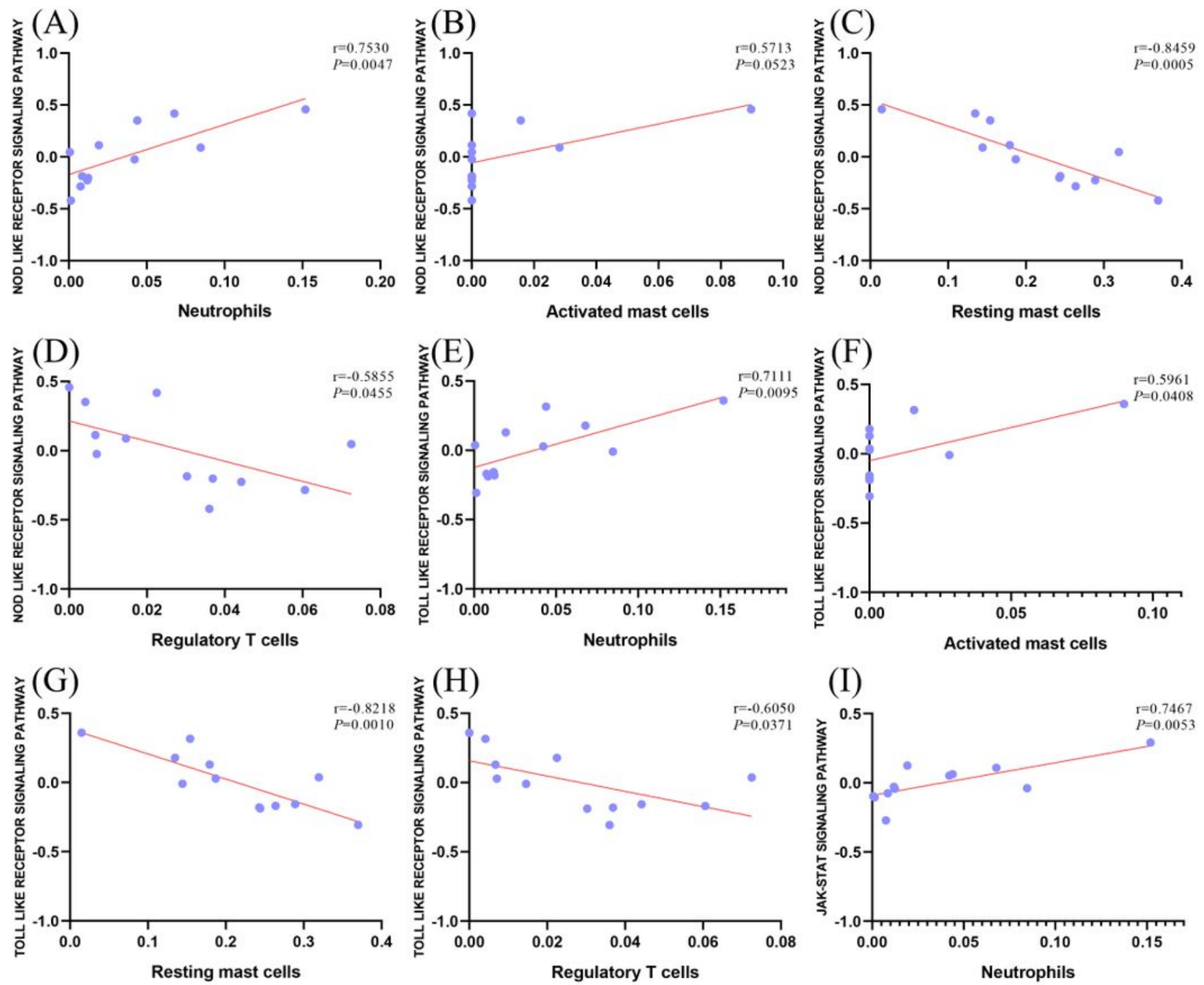

(J)

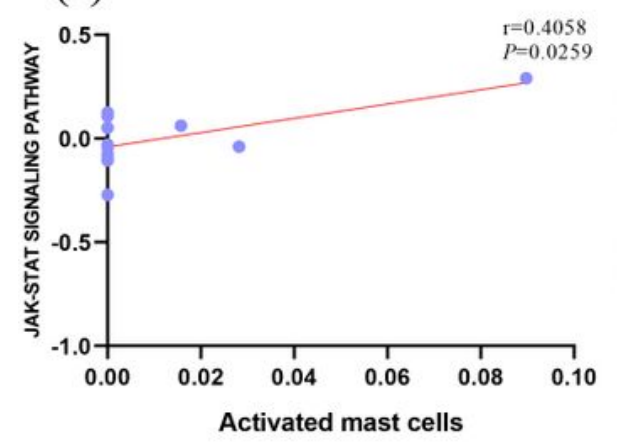

(K)

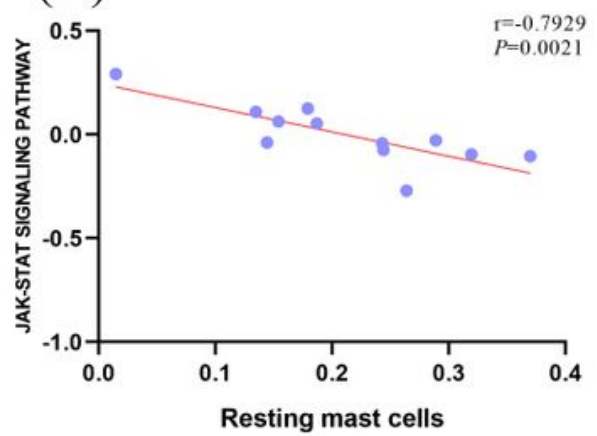

(L)

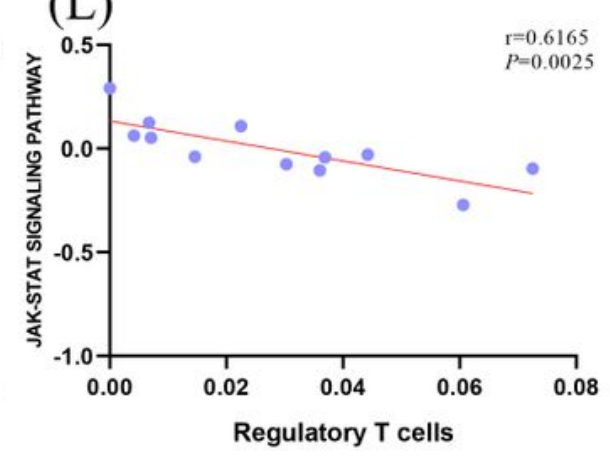

Figure 12

Infiltrating immune cells and critical pathways' correlation in the validation dataset. Neutrophils, activated mast cells were positively, resting mast cells and Tregs were negatively correlated with the JAK-STAT signaling pathway, NOD-like receptor signaling pathway, and Toll-like receptor signaling pathway (A-L). 


\section{Supplementary Files}

This is a list of supplementary files associated with this preprint. Click to download.

- Supplementarymaterials.docx 